\title{
$\mu$-norm and regularity
}

\author{
D.Treschev \\ Steklov Mathematical Institute of Russian Academy of Sciences
}

\begin{abstract}
In 22 we introduce the concept of a $\mu$-norm for a bounded operator in a Hilbert space. The main motivation is the extension of the measure entropy to the case of quantum systems. In this paper we recall the basic results from 22 and present further results on the $\mu$-norm. More precisely, we specify three classes of unitary operators for which the $\mu$-norm generates a bistochastic operator. We plan to use the latter in the construction of quantum entropy.
\end{abstract}

\section{Introduction}

Let $\mathcal{X}$ be a nonempty set and let $\mathcal{B}$ be a $\sigma$-algebra of subsets $X \subset \mathcal{X}$. Consider the measure space $(\mathcal{X}, \mathcal{B}, \mu)$, where $\mu$ is a probability measure: $\mu(\mathcal{X})=1$.

Consider the Hilbert space $\mathcal{H}=L^{2}(\mathcal{X}, \mu)$ with the scalar product and the norm

$$
\langle f, g\rangle=\int_{\mathcal{X}} f \bar{g} d \mu, \quad\|f\|=\sqrt{\langle f, f\rangle} .
$$

For any bounded operator $W$ on $\mathcal{H}$ let $\|W\|$ be its $L^{2}$ norm:

$$
\|W\|=\sup _{\|f\|=1}\|W f\| .
$$

We say that $\chi=\left\{Y_{1}, \ldots, Y_{J}\right\}$ is a (finite, measurable) partition (of $\mathcal{X}$ ) if

$Y_{j} \in \mathcal{B}, \quad \mu\left(\mathcal{X} \backslash \cup_{1 \leq j \leq J} Y_{j}\right)=0, \quad \mu\left(Y_{j} \cap Y_{k}\right)=0 \quad$ for any $j, k \in\{1, \ldots, J\}, k \neq j$.

We say that $\kappa=\left\{X_{1}, \ldots, X_{K}\right\}$ is a subpartition of $\chi=\left\{Y_{1}, \ldots, Y_{J}\right\}$ if for any $k \in$ $\{1, \ldots, K\}$ there exists $j \in\{1, \ldots, J\}$ such that $\mu\left(X_{k} \backslash Y_{j}\right)=0$.

For any $X \in \mathcal{B}$ consider the orthogonal projector

$$
\pi_{X}: \mathcal{H} \rightarrow \mathcal{H}, \quad \mathcal{H} \ni f \mapsto \pi_{X} f=\mathbf{1}_{X} \cdot f,
$$

where $\mathbf{1}_{X}$ is the indicator of $X$.

Let $W$ be a bounded operator on $\mathcal{H}$. For any partition $\chi=\left\{Y_{1}, \ldots, Y_{J}\right\}$ we define

$$
\mathcal{M}_{\chi}(W)=\sum_{j=1}^{J} \mu\left(Y_{j}\right)\left\|W \pi_{Y_{j}}\right\|^{2} .
$$


In [22] we have introduced the definition of the $\mu$-norm:1

$$
\|W\|_{\mu}=\inf _{\chi} \sqrt{\mathcal{M}_{\chi}(W)} .
$$

Recall that the operator $U$ is said to be an isometry if

$$
\langle f, g\rangle=\langle U f, U g\rangle, \quad f, g \in \mathcal{H} .
$$

If the isometry $U$ is invertible then $U$ is a unitary operator.

For any bounded $W$, any $Y \in \mathcal{B}$, and any isometry $U$

$$
\left\|W \pi_{Y}\right\| \leq\|W\|, \quad\|U W\|=\|W\|, \quad\left\|\pi_{Y}\right\|=1(\text { if } \mu(Y)>0) .
$$

This implies the following obvious properties of the $\mu$-norm:

$$
\begin{aligned}
\|\mathrm{id}\|_{\mu} & =1, \quad\|W\|_{\mu} \leq\|W\|, \\
\left\|W_{1} W_{2}\right\|_{\mu} & \leq\left\|W_{1}\right\|\left\|W_{2}\right\|_{\mu}, \\
\|\lambda W\|_{\mu} & =|\lambda|\|W\|_{\mu} \quad \text { for any } \lambda \in \mathbb{C}, \\
\|W\|_{\mu} & =\|U W\|_{\mu} \quad \text { for any isometry } U .
\end{aligned}
$$

The $\mu$-norm is motivated by the problem of the extension of the measure entropy to the case of quantum systems. Now we describe briefly the idea.

Let $F: \mathcal{X} \rightarrow \mathcal{X}$ be an endomorphism of the probability space $(\mathcal{X}, \mathcal{B}, \mu)$. This means that for any $X \in \mathcal{B}$ the set $F^{-1}(X)$ (the complete preimage) also lies in $\mathcal{B}$ and $\mu(X)=$ $\mu\left(F^{-1}(X)\right)$. Invertible endomorphisms are called automorphisms. Let $\operatorname{End}(\mathcal{X})$ denote the semigroup of all endomorphisms of $(\mathcal{X}, \mathcal{B}, \mu)$. There are two standard constructions.

(1) Any $F \in \operatorname{End}(\mathcal{X}, \mu)$ generates the isometry (a unitary operator if $F$ is an automotphism) $U_{F}$ on $\mathcal{H}$ (the Koopman operator):

$$
L^{2}(\mathcal{X}, \mu) \ni f \mapsto U_{F} f=f \circ F, \quad U_{F}=: \operatorname{Koop}(F) .
$$

(2) For any $F \in \operatorname{End}(\mathcal{X}, \mu)$ it is possible to compute the measure entropy $h(F)$.

Our question is as follows. Is it possible to determine in some "natural way" a real nonnegative function $\mathfrak{h}$ on the semigroup of isometries $\operatorname{Iso}(\mathcal{H})$ so that the diagram

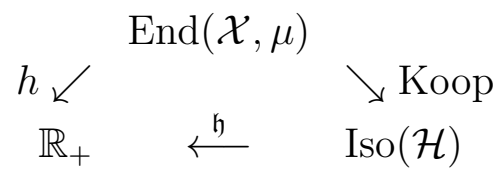

is commutative?

Recall the construction of the measure entropy of an endomorphism. Let $J_{N}$ be the set of multiindices $j=\left(j_{0}, \ldots, j_{N}\right)$, where any component $j_{n}$ takes values in the set $\{0, \ldots, K\}$. For any partition $\chi=\left\{X_{0}, \ldots, X_{K}\right\}$ and $j \in J_{N}$ we define

$$
\mathbf{X}_{j}=F^{-N}\left(X_{j_{N}}\right) \cap \ldots \cap F^{-1}\left(X_{j_{1}}\right) \cap X_{j_{0}} .
$$

\footnotetext{
${ }^{1}$ in fact, a seminorm

${ }^{2}$ known also as the Kolmogorov-Sinai entropy
} 
We define $h_{F}(\chi, N+1)$ by

$$
h_{F}(\chi, N+1)=-\sum_{j \in J_{N}} \mu\left(\mathbf{X}_{j}\right) \log \mu\left(\mathbf{X}_{j}\right)
$$

The function $h_{F}$, as a function of the second argument, is subadditive: $h_{F}(\chi, n+m) \leq$ $h_{F}(\chi, n)+h_{F}(\chi, m)$. This implies existence of the limit

$$
h_{F}(\chi)=\lim _{n \rightarrow \infty} \frac{1}{n} h_{F}(\chi, n) .
$$

Finally, the measure entropy is defined by

$$
h(F)=\sup _{\chi} h_{F}(\chi) .
$$

A rough idea is to construct the entropy of a unitary operator $U$ analogously with the following difference. Instead of $\mathbf{X}_{j}$ we take

$$
\mathfrak{X}_{j}=\pi_{X_{j_{N}}} U \pi_{X_{j_{N-1}}} U \ldots U \pi_{X_{j_{1}}} U \pi_{X_{j_{0}}} .
$$

We define

$$
\mathfrak{h}_{U}(\chi, N+1)=-\sum_{j \in J_{N}}\left\|\mathfrak{X}_{j}\right\|_{\mu}^{2} \log \left\|\mathfrak{X}_{j}\right\|_{\mu}^{2} .
$$

Other details are the same:

$$
\mathfrak{h}_{U}(\chi)=\lim _{n \rightarrow \infty} \frac{1}{n} \mathfrak{h}(\chi, n), \quad \mathfrak{h}(U)=\sup _{\chi} \mathfrak{h}_{U}(\chi)
$$

(provided the limit (1.10) exists).

In 22] we prove that for any automorphism $F$

$$
\mathfrak{h}\left(U_{F}\right)=h(F) \text {. }
$$

In the literature there exist several attempts to extend the concept of the measure entropy to quantum systems, see [6, 17, 18, 2, 3, 15, 13] and many others. In [1] several mutual relations between these approaches are given. Some works (for example, [2, 21, 19, 4, 14]) deal with the finite-dimensional case $(\# \mathcal{X}<\infty)$. In [8, 9] a construction for the measure entropy is proposed for doubly stochastic (bistochastic) operators on various spaces of functions on a measure space. It remains unclear, which approach to quantum generalization of the measure entropy is "more physical". This issue may become more clear after computation of the entropy $\mathfrak{h}$ or its analogs in examples. We plan to do this in forthcoming papers.

The above definition of $\mathfrak{h}(U)$ meets several technical problems, including the question on subadditivity 3 of $\mathfrak{h}_{U}(\chi, n)$ and the inequality $\mathfrak{h}_{U}(\chi) \leq \mathfrak{h}_{U}(\kappa)$ if $\kappa$ is a subpartition of $\chi 4$. We plan to change slightly the definition by replacing $\left\|\mathfrak{X}_{j}\right\|_{\mu}^{2}$ in (1.9) by another

\footnotetext{
${ }^{3}$ This subadditivity is important for the existence of the limit (1.10).

${ }^{4}$ This inequality is necessary if we want to approach the supremum (1.10) on fine partitions $\chi$.
} 
quantity similar to it to satisfy these two properties. To this end we associate with $U$ a bistochastic operator on $L^{1}(\mathcal{X}, \mu)$. Then the entropy of $U$ may be defined analogously to [8, 9]. We will present details in another paper, but seems, this bistochastic operator may turn to be interesting by itself. As we will see below in this paper, the concept of the $\mu$-norm remains central in all our constructions.

We use the $\mu$-norm to construct a bistochastic operator in three cases: Koopman operators, the case $\# \mathcal{X}<\infty$ and regular operators (a special class of operators defined in Section 4) for $\mathcal{X}=\mathbb{T}$. It is more or less clear that the circle $\mathbb{T}$ may be replaced by the torus $\mathbb{T}^{d}$, but this will be a subject of another paper.

Before systematic attempts to compute entropy of various unitary operators and to study its proprties we have to study the $\mu$-norm $\|\cdot\|_{\mu}$. We have started this in [22]. The present paper is a continuation of this program.

\section{Notation and previous results}

Here we collect basic results from [22]. We will refer to some of them below.

(1). $\left\|\pi_{X}\right\|_{\mu}^{2}=\mu(X)$ for any $X \in \mathcal{B}$.

(2). If $\chi^{\prime}$ is a subpartition of $\chi$ then $\mathcal{M}_{\chi^{\prime}}(W) \leq \mathcal{M}_{\chi}(W)$. Hence the quantities $\mathcal{M}_{\chi}(W)$ approach the infinum (1.3) on fine (small scale) partitions.

(3). For any two bounded operators $W_{1}$ and $W_{2}$

$$
\left\|W_{1}+W_{2}\right\|_{\mu} \leq\left\|W_{1}\right\|_{\mu}+\left\|W_{2}\right\|_{\mu} .
$$

This triangle inequality combined with (1.6) imply that $\|\cdot\|_{\mu}$ is a seminorm on the space of bounded operators on $\mathcal{H}$.

(4). Let $F$ be an automorphism of $(\mathcal{X}, \mathcal{B}, \mu)$ and let $U_{F}=\operatorname{Koop}(F)$. Then

$$
U_{F} \pi_{X}=\pi_{F^{-1}(X)} U_{F} \quad \text { for any } X \in \mathcal{B} .
$$

For any bounded operator $W$

$$
\left\|W U_{F}\right\|_{\mu}=\|W\|_{\mu} .
$$

This implies $\left\|U_{F}^{-1} W U_{F}\right\|_{\mu}=\|W\|_{\mu}$. Informally speaking, this means that measure preserving coordinate changes on $\mathcal{X}$ preserve the $\mu$-norm.

(5). $\|\cdot\|_{\mu}$ is a continuous function in the $L^{2}(\mathcal{X}, \mu)$ operator topology.

(6). If the measure $\mu$ has no atoms then $\left\|W+W_{0}\right\|_{\mu}=\|W\|_{\mu}$ for any bounded $W$ and compact $W_{0}$. In particular, $\mu$-norm of any compact operator vanishes. In fact, there exist non-compact operators with zero $\mu$-norm.

(7). Given $g \in L^{\infty}(\mathcal{X}, \mu)$ let $\widehat{g}$ be the multiplication operator defined by $f \mapsto \widehat{g} f=g f$. Then $\|\widehat{g}\|_{\mu}=\|g\|$.

(8). Suppose $\mathcal{X}=\{1, \ldots, J\}$ is finite and the measure of any element equals $1 / J$. Then $\mathcal{H}$ is isomorphic to $\mathbb{C}^{J}$ with the Hermitian product $\langle f, g\rangle_{J}=\frac{1}{J} \sum_{j=1}^{J} f(j) \overline{g(j)}$. Let

$$
f \mapsto W f, \quad(W f)(k)=\sum_{j=1}^{J} W(k, j) f(j)
$$


be an operator on $\mathcal{H}$. Then

$$
\|W\|_{\mu}^{2}=\frac{1}{J} \sum_{j, k=1}^{J}|W(k, j)|^{2}
$$

(9). For any partition $\left\{X_{1}, \ldots, X_{K}\right\}$ of $\mathcal{X}$

$$
\|W\|_{\mu}^{2}=\sum_{k=1}^{K}\left\|W \pi_{X_{k}}\right\|_{\mu}^{2}, \quad\|W\|_{\mu}^{2} \leq \sum_{k=1}^{K}\left\|\pi_{X_{k}} W\right\|_{\mu}^{2}
$$

(10). Let $\mathcal{X}$ be a compact metric space and $\mu$ a Borel measure w.r.t. the corresponding topology. Let $B_{r}(x) \subset \mathcal{X}$ denote the open ball with center at $x$ and radius $r$. Then for any $x \in \mathcal{X}$ the limit

$$
\vartheta(x)=\lim _{\varepsilon \searrow 0}\left\|W \pi_{B_{\varepsilon}(x)}\right\|^{2}
$$

exists, the function $\vartheta$ is measurable and $\|W\|_{\mu}^{2} \leq \int_{\mathcal{X}} \vartheta d \mu$. There exists an example which shows that in general this inequality is strict. However $\|W\|_{\mu}^{2}=\int_{\mathcal{X}} \vartheta d \mu$ provided two additional conditions $\mathbf{C 1}$ and $\mathbf{C 2}$ hold:

C1. The function $\vartheta$ is continuous.

C2. There exists $c>0$ such that for any open $\mathcal{O} \subset \mathcal{X}$, $\operatorname{diam}(\mathcal{O}) \leq \varepsilon$ and any $x \in \mathcal{O}$ there exists a function $f=\pi_{\mathcal{O}} f$ satisfying

$$
\left|\|W f\|^{2}-\vartheta(x)\|f\|^{2}\right| \leq \gamma(\varepsilon)\|f\|^{2}, \quad c<|f|_{\mathcal{O}} \mid<c^{-1},
$$

where $\gamma(\varepsilon) \rightarrow 0$ as $\varepsilon \rightarrow 0$. As usual, $\left.f\right|_{\mathcal{O}}$ denotes restriction of $f$ to the set $\mathcal{O}$.

The further results from [22] we mention here concern the case $\mathcal{X}=\mathbb{T}=\mathbb{R} / 2 \pi \mathbb{Z}$ with the Lebesgue measure $\mu$. We expect that they can be extended to the case $\mathcal{X}=\mathbb{T}^{d}, d>1$.

(11). Let $\mathcal{X}=\mathbb{T}$ be a circle with the Lebesgue measure $d \mu=\frac{1}{2 \pi} d x$. For any bounded sequence $\left\{\lambda_{k}\right\}_{k \in \mathbb{Z}}$ we consider the distribution $\lambda(x)=\sum \lambda_{k} e^{i k x}$. The convolution operator

$$
f \mapsto \operatorname{Conv}_{\lambda} f=\lambda * f:=\int_{\mathbb{T}} \lambda(y) f(\cdot-y) d y
$$

is bounded: $\left\|\operatorname{Conv}_{\lambda}\right\|=\sup _{k \in \mathbb{Z}}\left|\lambda_{k}\right|$. Then

$$
\left\|\operatorname{Conv}_{\lambda}\right\|_{\mu}^{2}=\rho(\lambda), \quad \rho(\lambda)=\limsup _{\# I \rightarrow \infty} \rho_{I}(\lambda), \quad \rho_{I}(\lambda)=\frac{1}{\# I} \sum_{k \in I}\left|\lambda_{k}\right|^{2},
$$

where $I \subset \mathbb{Z}$ are integer intervals.

(12). One of the main technical tools in the analysis of the $\mu$-norm in the case $\mathcal{X}=\mathbb{T}$ is the following lemma on Fourier coefficients of localized functions on the circle. 
Lemma 2.1 Let $Y=[a-\varepsilon, a+\varepsilon]$ and $f=\pi_{Y} f=\sum_{k \in \mathbb{Z}} f_{k} e^{i k x} \in L^{2}(\mathbb{T})$. Then for any integer interval $J$ and any $m \in \mathbb{Z}$

$$
\begin{aligned}
\left\|f-e^{i m(x-a)} f\right\| & \leq|m| \varepsilon\|f\|, \\
\left|f_{m}-e^{i l a} f_{m+l}\right| & \leq \frac{\varepsilon^{3 / 2}}{\sqrt{\pi}}|l|\|f\|, \\
\left|\sum_{k \in \mathbb{Z}} e^{-i m a} f_{k} \bar{f}_{k+m}-\|f\|^{2}\right| & \leq|m| \varepsilon\|f\|^{2} .
\end{aligned}
$$

(13). Consider the operator $W=\left(W_{j, k}\right)_{j, k \in \mathbb{Z}^{d}}$ on $\mathcal{H}=L^{2}(\mathbb{T})$ :

$$
f=\sum_{k \in \mathbb{Z}} f_{k} e^{i k x} \mapsto W f=\sum_{j, k \in \mathbb{Z}} W_{j, k} f_{k} e^{i j x}
$$

We say that $W$ is of diagonal type $(W \in \mathcal{D} \mathcal{T}(\mathbb{T}))$ if

$$
\sup _{j \in \mathbb{Z}}\left|W_{j+k, j}\right|=c_{k}<\infty, \quad k \in \mathbb{Z} \quad \text { and } \quad \sum_{k \in \mathbb{Z}} c_{k}=\mathbf{c}<\infty .
$$

The sequence $c_{s}$ is said to be the majorating sequence for $W \in \mathcal{D} \mathcal{T}(\mathbb{T})$. We define the norm $\|W\|_{\mathcal{D} \mathcal{T}}=\mathbf{c}$.

Operators from $\mathcal{D} \mathcal{T}(\mathbb{T})$ are bounded. As simple examples we have the following operators of diagonal type.

(a) Bounded convolution operators.

(b) Operators of multiplication by functions with absolutely converging Fourier series.

(c) The conjugated operator $W^{*}$ if $W \in \mathcal{D} \mathcal{T}(\mathbb{T})$.

(d) Linear combinations and products of operators of diagonal type. Moreover,

$$
\left\|W^{\prime} W^{\prime \prime}\right\|_{\mathcal{D} \mathcal{T}} \leq\left\|W^{\prime}\right\|_{\mathcal{D} \mathcal{T}}\left\|W^{\prime \prime}\right\|_{\mathcal{D} \mathcal{T}} \quad \text { for all } W^{\prime}, W^{\prime \prime} \in \mathcal{D} \mathcal{T}(\mathbb{T})
$$

(14). We prove that the normed space $\left(\mathcal{D T}(\mathbb{T}),\|\cdot\|_{\mathcal{D T}}\right)$ is closed. As a corollary we obtain that $\left(\mathcal{D T}(\mathbb{T}),\|\cdot\|_{\mathcal{D} \mathcal{T}}\right)$ is a $C^{*}$-algebra.

(15). We have the following inequalities between the norms:

$$
\begin{gathered}
\|W\|_{\mu} \leq\|W\| \leq\|W\|_{\mathcal{D} \mathcal{T}} \text { for any } W \in \mathcal{D} \mathcal{T}(\mathbb{T}) \\
\|f\|_{\infty} \leq\|\widehat{f}\|_{\mathcal{D} \mathcal{T}} \text { for any } f \text { with absolutely converging Fourier series, }
\end{gathered}
$$

where $\widehat{f}$ is the operator of multiplication by $f$.

(16). We associate with $W \in \mathcal{D} \mathcal{T}(\mathbb{T})$ and any point $a \in \mathbb{T}$ the distribution $L_{a}$,

$$
L_{a}=\sum_{j \in \mathbb{Z}} w_{j}(a) e^{i j x}, \quad w_{j}(a)=\sum_{k \in \mathbb{Z}} W_{j, k} e^{i(j-k) a} .
$$

For any $l \in \mathbb{Z}$ and $a \in \mathbb{T}$ we have the estimate

$$
\left|w_{l}(a)\right| \leq \mathbf{c}=\|W\|_{\mathcal{D} \mathcal{T}}
$$


We prove that for any $W \in \mathcal{D} \mathcal{T}(\mathbb{T})$ the function $a \mapsto \rho\left(L_{a}\right)=\limsup _{\# I \rightarrow \infty} \rho_{I}\left(L_{a}\right)$ $\left(I \subset \mathbb{Z}\right.$ are intervals, $\rho_{I}$ is defined in item (11) $)$ is continuous and

$$
\|W\|_{\mu}^{2}=\frac{1}{2 \pi} \int_{\mathbb{T}} \rho\left(L_{a}\right) d a
$$

(17). For any operator $W \in \mathcal{D} \mathcal{T}(\mathbb{T})$ we introduce the average trace of $W^{*} W$ by

$$
\mathbf{T}(W)=\limsup _{\# I \rightarrow \infty} \frac{1}{\# I} \sum_{j \in \mathbb{Z}, l \in I}\left|W_{l, j}\right|^{2},
$$

where $I \subset \mathbb{Z}$ are intervals. Then

$$
\mathbf{T}(W) \leq\|W\|_{\mu}^{2}
$$

(18). We also prove that if $U \in \mathcal{D} \mathcal{T}(\mathbb{T})$ is a unitary operator then

$$
\mathbf{T}(W)=\mathbf{T}(W U)=\mathbf{T}(U W) .
$$

\section{Main results}

In this section we collect main results of the present paper. In short, these results concern (1) properties of the $\mu$-norm on $\mathcal{R}(\mathbb{T})$, a special class of operators on $L^{2}(\mathbb{T})$, and (2) a construction of a bistochastic operator $\mathcal{W}$ on $L^{1}(\mathcal{X}, \mu)$ associated with an operator $W$ under some conditions, imposed on $W$.

- We say that an operator $W \in \mathcal{D} \mathcal{T}(\mathbb{T})$ is regular (the notation is $W \in \mathcal{R}(\mathbb{T})$ ) if for any $m, n \in \mathbb{Z}$ there exists the limit

$$
\omega_{m, n}=\lim _{\# I \rightarrow \infty} \omega_{I, m, n}, \quad \omega_{I, m, n}=\frac{1}{\# I} \sum_{j \in \mathbb{Z}, l \in I} W_{l+m, j} \bar{W}_{l, j+n} .
$$

- By Lemma $4.2 \mathcal{R}(\mathbb{T})$ is a cone closed with respect to the norm $\|\cdot\|_{\mathcal{D} \mathcal{T}}$.

- By Lemma $4.3\|W\|_{\mu}^{2}=\mathbf{T}(W)$ for any $W \in \mathcal{R}(\mathbb{T})$ (compare with (2.12) ).

- Let $\mathcal{A C F}(\mathbb{T})$ be the space of functions on $\mathbb{T}$ with absolutely converging Fourier series. Then the space of multiplication operators $\widehat{g}$ by functions $g \in \mathcal{A C F}(\mathbb{T})$ form a $C^{*}$-subalgebra in the $C^{*}$-algebra $\mathcal{D} \mathcal{T}(\mathbb{T})$.

- In Section 5 we present several examples of regular operators.

Let $W \in \mathcal{D} \mathcal{T}(\mathbb{T})$ be an operator with periodic matrix i.e., there exists $\tau \in \mathbb{N}$ such that $W_{j+\tau, k+\tau}=W_{j, k}$ for any $j, k \in \mathbb{Z}$. Any such operator is regular (Lemma 5.1). Another example of a regular operator is $\operatorname{Conv}_{\lambda}$, where $\lambda_{k}=e^{i \tau k^{2}}$.

- By Proposition 6.1 for any $W \in \mathcal{R}(\mathbb{T})$ and $g_{1}, g_{2} \in \mathcal{A C F}(\mathbb{T})$ the operator $\widehat{g}_{1} W \widehat{g}_{2}$ is regular. 
- In Section 6 we associate with operators $W$ from the following three classes

(1) $\operatorname{Koopman}$ operators $\operatorname{Koop}(F), F \in \operatorname{Aut}(\mathcal{X}, \mu)$,

(2) operators in the case $\# \mathcal{X}<\infty$,

(3) operators from $\mathcal{R}(\mathbb{T})$

a measure $d \mu_{W}\left(x^{\prime}, x^{\prime \prime}\right)=\nu\left(x^{\prime}, x^{\prime \prime}\right) d \mu\left(x^{\prime}\right) d \mu\left(x^{\prime \prime}\right)$ on $\mathcal{X} \times \mathcal{X}$ such that for any "sufficiently regular" functions $g^{\prime}, g^{\prime \prime}: \mathcal{X} \rightarrow \mathbb{C}$

$$
\left\|\widehat{g}^{\prime} W \widehat{g}^{\prime \prime}\right\|_{\mu}^{2}=\int_{\mathcal{X}^{2}}\left|g^{\prime}\left(x^{\prime}\right)\right|^{2}\left|g^{\prime \prime}\left(x^{\prime \prime}\right)\right|^{2} d \mu_{W}\left(x^{\prime}, x^{\prime \prime}\right) .
$$

- In Section 7 we introduce the operator

$$
L^{1}(\mathcal{X}, \mu) \ni f \mapsto \mathcal{W} f=\int_{\mathcal{X}} \nu(\cdot, a) f(a) d \mu(a)
$$

and prove (Lemma 7.1) that $\mathcal{W}$ is a bistochastic operator on $L^{1}(\mathcal{X}, \mu)$.

\section{Regular operators}

\subsection{Definition of $\omega_{m, n}$}

Definition 4.1 We say that $W \in \mathcal{D} \mathcal{T}(\mathbb{T})$ is regular $(W \in \mathcal{R}(\mathbb{T})$ ) if for any $m, n \in \mathbb{Z}$ there exists the limit

$$
\lim _{\# I \rightarrow \infty} \omega_{I, m, n}=\omega_{m, n}, \quad \omega_{I, m, n}=\frac{1}{\# I} \sum_{j \in \mathbb{Z}, l \in I} W_{l+m, j} \bar{W}_{l, j+n},
$$

where I are integer intervals.

Note that $\omega_{0,0}$ coincides with the average trace of $W^{*} W$ :

$$
\omega_{0,0}=\mathbf{T}(W) \quad \text { if } W \in \mathcal{R}(\mathbb{T}) .
$$

For any integer interval $I$ we put

$$
v_{I, m}(a)=\sum_{l \in I} \frac{w_{l+m}(a) \bar{w}_{l}(a)}{\# I}
$$

where the functions $w_{l}(a)$ are defined in (2.9).

Lemma 4.1 Suppose $W$ is regular. Then for any $m \in \mathbb{Z}$ there exists the limit

$$
\lim _{\# I \rightarrow \infty} v_{I, m}(a)=v_{m}(a), \quad v_{m}(a)=\sum_{n \in \mathbb{Z}} \omega_{m, n} e^{i(m+n) a}
$$

uniformly in $a \in \mathbb{T}$. The Fourier series of the function $v_{m}$ absolutely converges. 
Proof. By (2.9)

$$
\begin{aligned}
v_{m}(a) & =\lim _{\# I \rightarrow \infty} \frac{1}{\# I} \sum_{j, k \in \mathbb{Z}, l \in I} W_{l+m, j} \bar{W}_{l, k} e^{i(m-j+k) a} \\
& =\lim _{\# I \rightarrow \infty} \sum_{n \in \mathbb{Z}} \omega_{I, m, n} e^{i(m+n) a}=\sum_{n \in \mathbb{Z}} \omega_{m, n} e^{i(m+n) a} .
\end{aligned}
$$

By (10.1) the limit is uniform in $a$. By (10.2) this Fourier series absolutely converges.

Note that by (2.10) for any interval $I \subset \mathbb{Z}$, any $m \in \mathbb{Z}$, and any $a \in \mathbb{T}$

$$
\left|v_{I, m}(a)\right| \leq \mathbf{c}^{2}, \quad\left|v_{m}(a)\right| \leq \mathbf{c}^{2} .
$$

\subsection{Closeness with respect to $\|\cdot\|_{\mathcal{D} \mathcal{T}}$}

If $W \in \mathcal{R}(\mathbb{T})$ then for any $\lambda \in \mathbb{C}$ the operator $\lambda W$ is also regular. Hence, regular operators form a cone $\mathcal{R}(\mathbb{T}) \subset \mathcal{D} \mathcal{T}(\mathbb{T})$.

Lemma 4.2 The cone $\mathcal{R}(\mathbb{T})$ is closed with respect to the norm $\|\cdot\|_{\mathcal{D} \mathcal{T}}$.

Proof. Suppose $\left\{W_{p}\right\}_{p \in \mathbb{N}}, W_{p} \in \mathcal{R}(\mathbb{T})$ is a Cauchy sequence. By (14) there exists $W=\lim _{p \rightarrow \infty} W_{p}$, where the limit is taken with respect to the norm $\|\cdot\|_{\mathcal{D} \mathcal{T}}$.

For any $\varepsilon>0$ there exists positive $N$ such that

$$
\text { for any integer } p, q>N \text { we have: }\left\|W_{p}-W_{q}\right\|_{\mathcal{D} \mathcal{T}}<\varepsilon \text {. }
$$

We define $\left(\omega_{p}\right)_{I, m, n}$ and $\left(\omega_{p}\right)_{m, n}$ by (4.1), where $W$ is replaced by $W_{p}$. Then

$$
\begin{aligned}
\left|\left(\omega_{p}\right)_{I, m, n}-\left(\omega_{q}\right)_{I, m, n}\right| & =\frac{1}{\# I}\left|\sum_{j \in \mathbb{Z}, l \in I}\left(\left(W_{p}\right)_{l+m, j}\left(\bar{W}_{p}\right)_{l, j+n}-\left(W_{q}\right)_{l+m, j}\left(\bar{W}_{q}\right)_{l, j+n}\right)\right| \\
& \leq \frac{1}{\# I}\left(\Sigma_{1}+\Sigma_{2}\right), \\
\Sigma_{1} & =\sum_{j \in \mathbb{Z}, l \in I}\left|\left(W_{p}\right)_{l+m, j}\left(\left(\bar{W}_{p}\right)_{l, j+n}-\left(\bar{W}_{q}\right)_{l, j+n}\right)\right|, \\
\Sigma_{2} & =\sum_{j \in \mathbb{Z}, l \in I}\left|\left(\left(W_{p}\right)_{l+m, j}-\left(W_{q}\right)_{l+m, j}\right)\left(\bar{W}_{q}\right)_{l, j+n}\right| .
\end{aligned}
$$

To estimate the sums $\Sigma_{1}$ and $\Sigma_{2}$, we put

$$
\left(c_{p}\right)_{k}=\sup _{j \in \mathbb{Z}}\left|\left(W_{p}\right)_{k+j, j}\right|, \quad\left(c_{q}\right)_{k}=\sup _{j \in \mathbb{Z}}\left|\left(W_{q}\right)_{k+j, j}\right|, \quad d_{k}=\sup _{j \in \mathbb{Z}}\left|\left(W_{p}\right)_{k+j, j}-\left(W_{q}\right)_{k+j, j}\right| .
$$

The sums $\sum_{k}\left(c_{p}\right)_{k}$ are uniformly bounded:

$$
\sum_{k \in \mathbb{Z}}\left(c_{p}\right)_{k} \leq \tilde{\mathbf{c}}, \quad \sum_{k \in \mathbb{Z}}\left(c_{q}\right)_{k} \leq \tilde{\mathbf{c}} \quad \text { for some constant } \tilde{\mathbf{c}} .
$$


Moreover, by (4.6)

$$
\sum_{k \in \mathbb{Z}} d_{k}<\varepsilon
$$

By (4.7) and (4.8)

$$
\Sigma_{1} \leq \sum_{j \in \mathbb{Z}, l \in I}\left(c_{p}\right)_{l+m-j} d_{l-j-n} \leq \# I \tilde{\mathbf{c}} \varepsilon .
$$

Analogously $\Sigma_{2} \leq \# I \tilde{\mathbf{c}} \varepsilon$. This implies that for any interval $I$

$$
\left|\left(\omega_{p}\right)_{I, m, n}-\left(\omega_{q}\right)_{I, m, n}\right| \leq 2 \tilde{\mathbf{c}} \varepsilon .
$$

Hence, $\left|\left(\omega_{p}\right)_{m, n}-\left(\omega_{q}\right)_{m, n}\right| \leq 2 \tilde{\mathbf{c}} \varepsilon$ i.e., for any integer $m, n$ the sequence $\left(\omega_{p}\right)_{m, n}, p \in \mathbb{N}$ is a Cauchy sequence.

\section{$4.3 \mu$-norm of a regular operator}

Lemma 4.3 If $W \in \mathcal{R}(\mathbb{T})$ then (compare with (2.12))

$$
\|W\|_{\mu}^{2}=\mathbf{T}(W) .
$$

Proof. By (2.9), (2.11) and (4.3)

$$
\|W\|_{\mu}^{2}=\frac{1}{2 \pi} \int_{\mathbb{T}} \lim _{\# I \rightarrow \infty} v_{I, 0}(a) d a .
$$

By Lemma 4.1 the limit $v_{0}(a)=\lim _{\# I \rightarrow \infty} v_{I, 0}(a)$ exists for any $a \in \mathbb{T}$ and by (4.5) $\left|v_{I, 0}(a)\right| \leq \mathbf{c}^{2}$ for all $I$ and $a$. Therefore by the Lebesgue theorem on bounded convergence we may exchange the integration and the limit:

$$
\begin{aligned}
\|W\|_{\mu}^{2} & =\lim _{\# I \rightarrow \infty} \frac{1}{2 \pi} \int_{\mathbb{T}} v_{I, 0}(a) d a=\lim _{\# I \rightarrow \infty} \frac{1}{2 \pi} \int_{\mathbb{T}} \sum_{j, k \in \mathbb{Z}, l \in I} \frac{1}{\# I} W_{l, j} \bar{W}_{l, k} e^{i(k-j) a} d a \\
& =\lim _{\# I \rightarrow \infty} \sum_{j \in \mathbb{Z}, l \in I} \frac{1}{\# I} W_{l, j} \bar{W}_{l, j}=\omega_{0,0} .
\end{aligned}
$$

By (4.1) this implies (4.9).

Assertion (18) and Lemma 4.3 imply the following.

Corollary 4.1 Suppose $W, U \in \mathcal{D} \mathcal{T}(\mathbb{T})$, where $U$ is unitary and both $W$ and $U W U^{-1}$ are regular. Then by (18) and (1.7)

$$
\|W\|_{\mu}=\left\|U W U^{-1}\right\|_{\mu}=\left\|W U^{-1}\right\|_{\mu} .
$$




\section{Regular operators: examples}

Definition 5.1 We say that the matrix $\left(W_{k, j}\right)$ of the operator $W \in \mathcal{D} \mathcal{T}(\mathbb{T})$ is $\tau$-periodic, $\tau \in \mathbb{N}$, if

$$
W_{k+\tau, j+\tau}=W_{k, j} \quad \text { for any } k, j \in \mathbb{Z} \text {. }
$$

In particular, for any $g \in \mathcal{A C \mathcal { F }}(\mathbb{T})$ matrix of the operator $\widehat{g} \in \mathcal{D} \mathcal{T}(\mathbb{T})$ is 1 -periodic.

Lemma 5.1 Suppose $W \in \mathcal{D} \mathcal{T}(\mathbb{T})$ is an operator with $\tau$-periodic matrix, $\tau \in \mathbb{N}$. Then $W \in \mathcal{R}(\mathbb{T})$ and $\omega_{m, n}=\breve{\omega}_{m, n}$,

$$
\breve{\omega}_{m, n}=\frac{1}{\tau} \sum_{j \in \mathbb{Z}, l \in J} W_{l+m, j} \bar{W}_{l, j+n},
$$

where $J \subset \mathbb{Z}$ is any interval with $\# J=\tau$.

Proof. By $\tau$-periodicity $\breve{\omega}_{m, n}$ does not depend on $J$. For any $I=\{s, s+1, \ldots, s+K\}$ let $I^{\prime} \subset I$ be the maximal integer interval of the form $I^{\prime}=\{s, s+1, \ldots, s+q \tau-1\}$, $q \in \mathbb{Z}$. We put $I^{\prime \prime}=\{s+q \tau, s+q \tau+1, \ldots, s+K\}$. Then $\# I^{\prime \prime}<\tau, \omega_{I^{\prime}, m, n}=\breve{\omega}_{m, n}$ while $\omega_{I, m, n}-\omega_{I^{\prime}, m, n}=A_{1}+A_{2}$,

$$
A_{1}=\frac{1}{\# I} \sum_{l \in I^{\prime \prime} \backslash I^{\prime}} W_{l+m, j} \bar{W}_{l, j+n}, \quad A_{2}=\left(\frac{1}{\# I}-\frac{1}{\# I^{\prime}}\right) \sum_{l \in I^{\prime}} W_{l+m, j} \bar{W}_{l, j+n} .
$$

The inequalities

$$
\left|A_{1}\right| \leq \frac{\tau \mathbf{c}^{2}}{\# I}, \quad\left|A_{2}\right| \leq \frac{\tau \mathbf{c}^{2}}{\# I-\tau}
$$

imply the existence of the limit (4.1) and the equation $\omega_{m, n}=\breve{\omega}_{m, n}$.

Corollary 5.1 Consider the operator $\widehat{g}$, where $g \in \mathcal{A C F}(\mathbb{T})$. After simple calculations we obtain:

$$
\omega_{m, n}(\widehat{g})=\mathbf{g}_{m+n}, \quad g(x) \bar{g}(x)=\sum_{k \in \mathbb{Z}} \mathbf{g}_{k} e^{i k x},
$$

where the last equation is the definition of $\mathbf{g}_{k}$.

Lemma 5.2 Let $\left\{\lambda_{k}\right\}_{k \in \mathbb{Z}}$ be defined by $\lambda_{k}=e^{i \tau k^{2}}$. Then the operator $\operatorname{Conv}_{\lambda}$ is regular:

$$
\omega_{m, n}=\delta_{0, m+n} \delta_{\tau m, \pi \mathbb{Z}} e^{i \tau m^{2}}, \quad \delta_{\tau m, \pi \mathbb{Z}}= \begin{cases}1 & \text { if } \tau m / \pi \in \mathbb{Z}, \\ 0 & \text { if } \tau m / \pi \notin \mathbb{Z}\end{cases}
$$

Proof. In this case $W_{k, j}=\delta_{k j} e^{i \tau k^{2}}$ and

$$
\begin{aligned}
\omega_{I, m, n} & =\frac{1}{\# I} \sum_{j \in \mathbb{Z}, l \in I} \delta_{l+m, j} \delta_{l, j+n} e^{i \tau\left((l+m)^{2}-l^{2}\right.} \\
& =\frac{1}{\# I} \sum_{l \in I} \delta_{l, l+m+n} e^{i \tau m^{2}+2 i \tau m l}=\frac{1}{\# I} \delta_{0, m+n} e^{i \tau m^{2}} \sum_{l \in I} e^{2 i \tau m l} .
\end{aligned}
$$

Hence, if $\tau m / \pi \in \mathbb{Z}$ then $\omega_{m, n}=\delta_{0, m+n} e^{i \tau m^{2}}$. If $\tau m / \pi \notin \mathbb{Z}$ then $\omega_{m, n}=0$. 


\section{Measure associated with an operator}

\subsection{Koopman operator}

Let $(\mathcal{X}, \mathcal{B}, \mu)$ be a probability space.

Lemma 6.1 $F \in \operatorname{Aut}(\mathcal{X}, \mu), g_{0}, \ldots, g_{K} \in L^{\infty}(\mathcal{X}, \mu)$. Then

$$
\left\|\widehat{g}_{K} U_{F} \widehat{g}_{K-1} \ldots U_{F} \widehat{g}_{0}\right\|_{\mu}^{2}=\int_{\mathcal{X}}\left|g_{K} \circ F^{K}\right|^{2}\left|g_{K-1} \circ F^{K-1}\right|^{2} \ldots\left|g_{0}\right|^{2} d \mu
$$
that 5

Proof. Take a small constant $\sigma>0$ and consider the partition $\left\{X_{1}, \ldots, X_{J}\right\}$ such

$$
\left\|g_{k}-\varphi_{k}\right\|_{\infty}<\sigma\left\|g_{k}\right\|_{\infty}, \quad \varphi_{k}=\sum_{j=1}^{J} g_{k, j} \mathbf{1}_{X_{j}}, \quad\left\|\varphi_{k}\right\|_{\infty} \leq\left\|g_{k}\right\|_{\infty}, \quad k=0, \ldots, K .
$$

Here $g_{k, j} \in \mathbb{C}$ are some constants. We put $S=\widehat{\varphi}_{K} U_{F} \widehat{\varphi}_{K-1} \ldots U_{F} \widehat{\varphi}_{0}$. By the triangle inequality the quantity

$$
\left|\left\|\widehat{g}_{K} U_{F} \widehat{g}_{K-1} \ldots U_{F} \widehat{g}_{0}\right\|_{\mu}-\|S\|_{\mu}\right|
$$

does not exceed

$$
\begin{aligned}
& \left|\left\|\widehat{g}_{K} U_{F} \widehat{g}_{K-1} \ldots U_{F} \widehat{g}_{0}\right\|_{\mu}-\left\|\widehat{\varphi}_{K} U_{F} \widehat{g}_{K-1} \ldots U_{F} \widehat{g}_{0}\right\|_{\mu}\right| \\
+ & \left|\left\|\widehat{\varphi}_{K} U_{F} \widehat{g}_{K-1} \ldots U_{F} \widehat{g}_{0}\right\|_{\mu}-\left\|\widehat{\varphi}_{K} U_{F} \widehat{g}_{K-1} \ldots U_{F} \widehat{g}_{0}\right\|_{\mu}\right| \\
+ & \ldots+\left|\left\|\widehat{\varphi}_{K} U_{F} \widehat{\varphi}_{K-1} \ldots \widehat{\varphi}_{1} U_{F} \widehat{g}_{0}\right\|_{\mu}-\left\|\widehat{\varphi}_{K} U_{F} \widehat{\varphi}_{K-1} \ldots \widehat{\varphi}_{1} U_{F} \widehat{\varphi}_{0}\right\|_{\mu}\right| \\
\leq \quad & \left\|\left(\widehat{g}_{K}-\widehat{\varphi}_{K}\right) U_{F} \widehat{g}_{K-1} \ldots U_{F} \widehat{g}_{0}\right\|_{\mu}+\left\|\widehat{\varphi}_{K} U_{F}\left(\widehat{g}_{K-1}-\widehat{\varphi}_{K-1}\right) \ldots U_{F} \widehat{g}_{0}\right\|_{\mu} \\
+ & \ldots+\left\|\widehat{\varphi}_{K} U_{F} \widehat{\varphi}_{K-1} \ldots \widehat{\varphi}_{1} U_{F}\left(\widehat{g}_{0}-\widehat{\varphi}_{0}\right)\right\|_{\mu} \\
\leq \quad & (K+1) \sigma \prod_{0 \leq k \leq K}\left\|g_{k}\right\|_{\infty} .
\end{aligned}
$$

By (2.1)

$$
S=\sum_{j_{0}, \ldots, j_{K}} g_{K, j_{K}} g_{K-1, j_{K-1}} \ldots g_{0, j_{0}} \pi_{X_{j_{K}}} \pi_{F^{-1}\left(X_{j_{K-1}}\right)} \ldots \pi_{F^{-K}\left(X_{j_{0}}\right)} U_{F}^{K}
$$

We put $X_{j_{K}, \ldots, j_{0}}=X_{j_{K}} \cap F^{-1}\left(X_{j_{K-1}}\right) \cap \ldots \cap F^{-K}\left(X_{j_{0}}\right)$. Note that for any two sets $X^{\prime}=X_{j_{K}^{\prime}, \ldots, j_{0}^{\prime}}$ and $X^{\prime \prime}=X_{j_{K}^{\prime \prime}, \ldots, j_{0}^{\prime \prime}}$ we have: $\mu\left(X^{\prime} \cap X^{\prime \prime}\right)=0$ if the collections of indices $j_{K}^{\prime}, \ldots, j_{0}^{\prime}$ and $j_{K}^{\prime \prime}, \ldots, j_{0}^{\prime \prime}$ do not coincide. Then by (2.2) and (1) we obtain:

$$
\begin{aligned}
S & =\sum_{j_{0}, \ldots, j_{K}}\left|g_{K, j_{K}} g_{K-1, j_{K-1}} \ldots g_{0, j_{0}}\right|^{2}\left\|\pi_{X_{j_{K}, \ldots, j_{0}}}\right\|_{\mu}^{2} \\
& =\sum_{j_{0}, \ldots, j_{K}}\left|g_{K, j_{K}} g_{K-1, j_{K-1}} \ldots g_{0, j_{0}}\right|^{2} \mu\left(X_{j_{K}, \ldots, j_{0}}\right) .
\end{aligned}
$$

\footnotetext{
${ }^{5}$ We can use the same partition for all the functions $g_{k}$.
} 
Equation (6.2) implies

$$
\varphi_{k} \circ F^{k}=\sum_{j=1}^{J} g_{k, j} \mathbf{1}_{F^{-k}\left(X_{j}\right)}
$$

Hence (6.3) is an integral sum for the integral

$$
\int_{\mathcal{X}}\left|\varphi_{K} \circ F^{K}\right|^{2}\left|\varphi_{K-1} \circ F^{K-1}\right|^{2} \ldots\left|\varphi_{0}\right|^{2} d \mu .
$$

This integral differs from the integral (6.1) at most by $2 \sigma \prod_{0 \leq k \leq K}\left\|g_{k}\right\|_{\infty}^{2}$. Since $\sigma$ is arbitrarily small, we obtain equation (6.1).

Now suppose that $\mathcal{X}$ is in addition a topological space and $\mathcal{B}$ is the corresponding Borel $\sigma$-algebra. Consider the distribution $\delta \in\left(C\left(\mathcal{X}^{2}\right)\right)^{*}$ (a measure on $\mathcal{X}^{2}$ by the Riesz theorem) such that for any $\Phi \in C\left(\mathcal{X}^{2}\right)$

$$
\int_{\mathcal{X}^{2}} \delta\left(x^{\prime}, x^{\prime \prime}\right) \Phi\left(x^{\prime}, x^{\prime \prime}\right) d \mu\left(x^{\prime}\right) d \mu\left(x^{\prime \prime}\right)=\int_{\mathcal{X}} \Phi(x, x) d \mu(x) .
$$

Taking in (6.4) $\Phi\left(x^{\prime}, x^{\prime \prime}\right)=\varphi^{\prime}\left(x^{\prime}\right) \varphi^{\prime \prime}\left(x^{\prime \prime}\right)$, where $\varphi^{\prime}, \varphi^{\prime \prime}: \mathcal{X} \rightarrow \mathbb{C}$ are arbitrary continuous functions, we obtain:

$$
\int_{\mathcal{X}} \delta\left(x^{\prime}, x^{\prime \prime}\right) \varphi^{\prime}\left(x^{\prime}\right) d \mu\left(x^{\prime}\right)=\varphi^{\prime}\left(x^{\prime \prime}\right), \quad \int_{\mathcal{X}} \delta\left(x^{\prime}, x^{\prime \prime}\right) \varphi^{\prime \prime}\left(x^{\prime \prime}\right) d \mu\left(x^{\prime \prime}\right)=\varphi^{\prime \prime}\left(x^{\prime}\right) .
$$

Recall also that if $F \in \operatorname{End}(\mathcal{X}, \mu)$

$$
\int_{\mathcal{X}} f d \mu=\int_{\mathcal{X}} f \circ F d \mu \text { for any } f \in L^{1}(\mathcal{X}, \mu) .
$$

Then for any continuous $F \in \operatorname{Aut}(\mathcal{X}, \mu)$

$$
\delta\left(x^{\prime}, x^{\prime \prime}\right)=\delta\left(F\left(x^{\prime}\right), F\left(x^{\prime \prime}\right)\right) .
$$

Indeed, for any $\Phi \in C\left(\mathcal{X}^{2}\right)$ by (6.6)

$$
\begin{aligned}
& \int_{\mathcal{X}^{2}} \delta\left(F\left(x^{\prime}\right), F\left(x^{\prime \prime}\right)\right) \Phi\left(x^{\prime}, x^{\prime \prime}\right) d \mu\left(x^{\prime}\right) d \mu\left(x^{\prime \prime}\right) \\
= & \int_{\mathcal{X}^{2}} \delta\left(x^{\prime}, x^{\prime \prime}\right) \Phi\left(F^{-1}\left(x^{\prime}\right), F^{-1}\left(x^{\prime \prime}\right)\right) d \mu\left(x^{\prime}\right) d \mu\left(x^{\prime \prime}\right)=\int_{\mathcal{X}} \Phi(x, x) d \mu(x) .
\end{aligned}
$$

Lemma 6.2 Let $F \in \operatorname{Aut}(\mathcal{X}, \mu)$ be continuous and $g_{0}, \ldots, g_{K} \in C(\mathcal{X})$. Then

$$
\begin{aligned}
& \left\|\widehat{g}_{K} U_{F} \ldots \widehat{g}_{1} U_{F} \widehat{g}_{0}\right\|_{\mu}^{2} \\
= & \int_{\mathcal{X}^{K+1}}\left|g_{K}\left(x_{K}\right)\right|^{2} \delta\left(x_{K}, F\left(x_{K-1}\right)\right) \ldots\left|g_{1}\left(x_{1}\right)\right|^{2} \delta\left(x_{1}, F\left(x_{0}\right)\right)\left|g_{0}\left(x_{0}\right)\right|^{2} d \mu^{K+1}, \\
& \quad d \mu^{K+1}=d \mu\left(x_{K}\right) \ldots d \mu\left(x_{0}\right) .
\end{aligned}
$$


Proof. Let $I$ denote the integral (6.8). We use in this integral the change of coordinates

$$
x_{0}=x_{0}^{\prime}, \quad x_{1}=F\left(x_{1}^{\prime}\right), \quad \ldots \quad x_{K}=F^{K}\left(x_{K}^{\prime}\right), \quad d \mu^{\prime K+1}=d \mu\left(x_{K}^{\prime}\right) \ldots d \mu\left(x_{0}^{\prime}\right) .
$$

Then

$$
\begin{aligned}
I= & \int\left|g_{K} \circ F^{K}\left(x_{K}^{\prime}\right)\right|^{2} \delta\left(F^{K}\left(x_{K}^{\prime}\right), F^{K}\left(x_{K-1}^{\prime}\right)\right) \\
& \ldots\left|g_{1} \circ F\left(x_{1}^{\prime}\right)\right|^{2} \delta\left(F\left(x_{1}^{\prime}\right), F\left(x_{0}^{\prime}\right)\right)\left|g_{0}\left(x_{0}^{\prime}\right)\right|^{2} d \mu^{\prime K+1} \\
= & \int_{\mathcal{X}}\left|g_{K} \circ F^{K}\right|^{2} \ldots\left|g_{1} \circ F\right|^{2}\left|g_{0}\right|^{2} d \mu .
\end{aligned}
$$

It remains to use Lemma 6.1.

We associate with any $U_{F}=\operatorname{Koop}(F)$, where $F \in \operatorname{Aut}(\mathcal{X}, \mu)$ is continuous 6 the measure $\mu_{U_{F}}$ on $\mathcal{X}^{2}$ :

$$
d \mu_{U_{F}}\left(x^{\prime}, x^{\prime \prime}\right)=\delta\left(x^{\prime}, F\left(x^{\prime \prime}\right)\right) d \mu\left(x^{\prime}\right) d \mu\left(x^{\prime \prime}\right) .
$$

\subsection{The finite-dimensional case}

Let $\mathcal{X}=\{1, \ldots, J\}$ be a finite set. We identify $\mathcal{X}$ with $\mathbb{Z}_{J}=\mathbb{Z} / J \mathbb{Z}$, the cyclic additive group with $J$ elements 7 Let the measure $\mu$ of any point be equal to $1 / J$. Then $\mathcal{H}=$ $L^{2}(\mathcal{X}, \mu) \cong\left(\mathbb{C}^{J},\langle\rangle,\right)$, where $\langle$,$\rangle equals the standard Hermitian product divided by J$.

We put $\eta=e^{2 \pi i / J}$. Then $\eta^{J}=1$ and $\bar{\eta}=\eta^{-1}$. The space $\mathcal{H}$ may be identified with the space of "discrete trigonometric polynomials"

$$
f=f(x)=\sum_{j \in \mathbb{Z}_{J}} f_{j} \eta^{j x}, \quad f_{j} \in \mathbb{C}, \quad x \in \mathbb{Z}_{J} .
$$

This polynomial representation generates on $\mathcal{H}$ an operation of multiplication: for any two vectors $f^{\prime}=\sum f_{j}^{\prime} \eta^{j x}$ and $f^{\prime \prime}=\sum f_{j}^{\prime \prime} \eta^{j x}$

$$
f^{\prime} f^{\prime \prime}=f=\sum_{k \in \mathbb{Z}_{J}} f_{k} \eta^{k x}, \quad f_{k}=\sum_{j \in \mathbb{Z}_{J}} f_{k-j}^{\prime} f_{j}^{\prime \prime} .
$$

This product introduces on $\mathcal{H}$ the structure of a commutative ring. The structure of a Hilbert space is determined by

$$
\langle f, g\rangle=\frac{1}{J} \sum_{k \in \mathbb{Z}_{J}} f(k) \overline{g(k)}
$$

The coefficients $f_{k}$ and $f(k)$ are connected by the "discrete Fourier transform"

$$
f(k)=\sum_{j \in \mathbb{Z}_{J}} f_{j} \eta^{k j}, \quad f_{j}=\frac{1}{J} \sum_{k \in \mathbb{Z}_{J}} f(k) \eta^{-k j} .
$$

\footnotetext{
${ }^{6}$ We expect that the continuity is inessential here.

${ }^{7}$ Below we also use the structure of a commutative ring on $\mathbb{Z}_{J}$.
} 
For any $f$ satisfying (6.10) and any $A \subset \mathbb{Z}_{J}$ we put

$$
\int_{A} f(x) d \mu(x):=\frac{1}{J} \sum_{k \in A} f(k)=\frac{1}{J} \sum_{k \in A, j \in \mathbb{Z}_{J}} f_{j} \eta^{j k} .
$$

Then

$$
\int_{\mathcal{X}} f d \mu=f_{0}, \quad\langle f, g\rangle=\frac{1}{J} \int_{\mathcal{X}} f \bar{g} d \mu .
$$

Consider a linear operator

$$
f \mapsto W f, \quad(W f)_{k}=\sum_{j \in \mathbb{Z}_{J}} W_{k j} f_{j}
$$

In another basis it takes the form

$$
(W f)(k)=\sum_{j \in \mathbb{Z}_{J}} W(k, j) f(j) .
$$

Equations (6.11) imply

$$
W(m, n)=\frac{1}{J} \sum_{j, k \in \mathbb{Z}_{J}} \eta^{m k} W_{k j} \eta^{-j n}
$$

We define

$$
\omega_{m, n}=\frac{1}{J} \sum_{j, l \in \mathbb{Z}_{J}} W_{l+m, j} \bar{W}_{l, j+n}, \quad \nu(x, a)=\sum_{m, n \in \mathbb{Z}_{J}} \omega_{m, n} \eta^{m x} \eta^{n a}, \quad x, a \in \mathbb{Z}_{J} .
$$

Lemma 6.3 $\sum_{m, n \in \mathbb{Z}_{J}}|W(m, n)|^{2}=\sum_{j, k \in \mathbb{Z}_{J}}\left|W_{j, k}\right|^{2}$.

Proof. Direct computation with the help of (‥12) and the identity $\sum_{j \in \mathbb{Z}_{J}} \eta^{j k}=J \delta_{k, 0}$.

Corollary 6.1 By (2.3) and Lemma 6.3

$$
\|W\|_{\mu}^{2}=\omega_{0,0}=\int_{\mathcal{X}^{2}} \nu(x, a) d \mu(x) d \mu(a) .
$$

Lemma 6.4 For any $g^{\prime}, g^{\prime \prime} \in \mathcal{H}$ the operator $\widetilde{W}=\widehat{g}^{\prime} W \widehat{g}^{\prime \prime}$ generates the coefficients $\widetilde{\omega}_{m, n}$ such that

$$
\sum_{m, n \in \mathbb{Z}} \widetilde{\omega}_{m, n} \eta^{m x} \eta^{n a}=\left|g^{\prime}(x)\right|^{2} \nu(x, a)\left|g^{\prime \prime}(a)\right|^{2} .
$$

The measure $d \mu_{W}(x, a)=\nu(x, a) d \mu(x) d \mu(a)$ on $\mathcal{X}^{2}$ satisfies

$$
\left\|\widehat{g}^{\prime} W \widehat{g}^{\prime \prime}\right\|_{\mu}^{2}=\int_{\mathcal{X}^{2}}\left|g^{\prime}(x)\right|^{2}\left|g^{\prime \prime}(a)\right|^{2} d \mu_{W}(x, a) .
$$


Proof. We put

$$
g^{\prime}(x)=\sum g_{k}^{\prime} \eta^{k x}, \quad g^{\prime \prime}(x)=\sum g_{k}^{\prime \prime} \eta^{k x}, \quad\left|g^{\prime}(x)\right|^{2}=\sum \mathbf{g}_{k}^{\prime} \eta^{k x}, \quad\left|g^{\prime \prime}(x)\right|^{2}=\sum \mathbf{g}_{k}^{\prime \prime} \eta^{k x} .
$$

The equation $\widetilde{W}_{p, q}=\sum_{\alpha, \beta} g_{p-\alpha}^{\prime} W_{\alpha, \beta} g_{\beta-q}^{\prime \prime}$ implies

$$
\begin{aligned}
\widetilde{\omega}_{m, n} & =\frac{1}{J} \sum_{l, j, \alpha, \beta} g_{l+m-\alpha^{\prime}}^{\prime} W_{\alpha^{\prime}, \beta^{\prime}} g_{\beta^{\prime}-j}^{\prime \prime} \bar{g}_{l-\alpha^{\prime \prime}}^{\prime} \bar{W}_{\alpha^{\prime \prime}, \beta^{\prime \prime}} \bar{g}_{\beta^{\prime \prime}-j-n}^{\prime \prime} \\
& =\frac{1}{J} \sum_{\alpha, \beta} \mathbf{g}_{m-\alpha^{\prime}+\alpha^{\prime \prime}}^{\prime} W_{\alpha^{\prime}, \beta^{\prime}} \bar{W}_{\alpha^{\prime \prime}, \beta^{\prime \prime}} \mathbf{g}_{\beta^{\prime}-\beta^{\prime \prime}+n}^{\prime \prime} \\
& =\frac{1}{J} \sum_{p, q, \alpha^{\prime \prime}, \beta^{\prime}} \mathbf{g}_{m-p}^{\prime} W_{p+\alpha^{\prime \prime}, \beta^{\prime}} \bar{W}_{\alpha^{\prime \prime}, \beta^{\prime}-q} \mathbf{g}_{q+n}^{\prime \prime}=\sum_{p, q} \mathbf{g}_{m-p}^{\prime} \omega_{p,-q} \mathbf{g}_{q+n}^{\prime \prime}
\end{aligned}
$$

This implies (6.15). Equation (‥16) follows from (6.14).

We put

$$
c_{k}=\max _{j \in \mathbb{Z}_{J}}\left|W_{k+j, j}\right|, \quad\|W\|_{\mathcal{D} \mathcal{T}}=\sum_{k \in \mathbb{Z}_{J}} c_{k}
$$

Then

$$
\sum_{m \in \mathbb{Z}_{J}}\left|\omega_{m, n}\right| \leq\|W\|_{\mathcal{D} \mathcal{T}}^{2}, \quad \sum_{n \in \mathbb{Z}_{J}}\left|\omega_{m, n}\right| \leq\|W\|_{\mathcal{D} \mathcal{T}}^{2}
$$

Definition 6.1 We say that $f=\sum_{j \in \mathbb{Z}_{J}} f_{j} \eta^{j x}$ is nonnegative ( $f \geq 0$ ) if the numbers $f(x)$ are real and nonnegative for all $x \in \mathbb{Z}_{J}$.

Lemma 6.5 If $\mathcal{X}=\mathbb{Z}_{J}$ then the following three statements are equivalent.

(1) $f \geq 0$

(2) $f=g \bar{g}$ for some $g \in \mathcal{H}$,

(3) $f_{k}=\sum_{s \in \mathbb{Z}_{J}} g_{k+s} \bar{g}_{s}$.

Proof. Equivalence of statements (2) and (3) is obvious. Equivalence of (1) and (2) follows from two simple facts.

(a) For any $g^{\prime}, g^{\prime \prime} \in \mathcal{H}$ and any $x \in \mathbb{Z}_{J} \quad\left(g^{\prime} g^{\prime \prime}\right)(x)=g^{\prime}(x) g^{\prime \prime}(x)$.

(b) The numbers $f(x), x \in \mathbb{Z}_{J}$ determine uniquely $f \in \mathcal{H}$.

Lemma 6.6 Suppose $f \geq 0$ and $F(x)=\sum_{m, n \in \mathbb{Z}_{J}} \omega_{m,-n} f_{n} \eta^{m x}$. Then $F \geq 0$.

Proof. By Lemma 6.5 $f=g \bar{g}$ for some $g \in \mathcal{H}$. Then

$$
F(x)=\frac{1}{J} \sum_{j, l, n, s \in \mathbb{Z}_{J}} W_{l+m, j} \bar{W}_{l, j-n} g_{n+s} \bar{g}_{s} \eta^{m x} .
$$

By using the change of summation indices $(n, s) \mapsto(k, q), k=j-n, q=s-k$, we obtain:

$$
F(x)=\frac{1}{J} \sum_{j, l, k, q \in \mathbb{Z}_{J}} W_{l+m, j} g_{j+q} \bar{W}_{l, k} \bar{g}_{k+q} \eta^{m x}=\sum_{q \in \mathbb{Z}} F^{(q)}(x) \bar{F}^{(q)}(x),
$$


where

$$
F^{(q)}(x)=\sum_{j, l \in \mathbb{Z}_{J}} W_{l, j} g_{j+q} \eta^{l x}
$$

Hence, $F \geq 0$.

Now suppose $W$ is a unitary operator:

$$
\frac{1}{J} \sum_{l \in \mathbb{Z}_{J}} W_{l, j} \bar{W}_{l, k}=\delta_{j, k}, \quad j, k \in \mathbb{Z}_{J}
$$

We put $\mathbf{1}_{\mathcal{X}}=\sqrt{J} \in \mathcal{H}$. Hence,

$$
\mathbf{1}_{\mathcal{X}}(x)=\sum_{k \in \mathbb{Z}_{J}}\left(\mathbf{1}_{\mathcal{X}}\right)_{k} \eta^{k x}, \quad\left(\mathbf{1}_{\mathcal{X}}\right)_{k}=\sqrt{J} \delta_{k, 0}, \quad\left\langle\mathbf{1}_{\mathcal{X}}, \mathbf{1}_{\mathcal{X}}\right\rangle=1
$$

Lemma 6.7 Suppose $W$ is unitary. Then

$$
\sum_{n \in \mathbb{Z}_{J}} \omega_{m,-n}\left(\mathbf{1}_{\mathcal{X}}\right)_{n}=\left(\mathbf{1}_{\mathcal{X}}\right)_{m}, \quad \sum_{m \in \mathbb{Z}_{J}}\left(\mathbf{1}_{\mathcal{X}}\right)_{m} \omega_{-m, n}=\left(\mathbf{1}_{\mathcal{X}}\right)_{n}
$$

Proof. By (6.13) and (6.18)

$$
\sum_{n \in \mathbb{Z}_{J}} \omega_{m,-n}\left(\mathbf{1}_{\mathcal{X}}\right)_{n}=\sum_{j, l, n \in \mathbb{Z}_{J}} \frac{W_{l+m, j} \bar{W}_{l, j-n}}{J} \sqrt{J} \delta_{n, 0}=\sum_{j, l \in \mathbb{Z}_{J}} \frac{W_{l+m, j} \bar{W}_{l, j}}{\sqrt{J}}=\left(\mathbf{1}_{\mathcal{X}}\right)_{m}
$$

The second equation (6.19) can be obtained analogously.

\subsection{Measure associated with a regular operator}

We start from a simple remark. Take any $f \in L^{2}(\mathbb{T})$. Then $f \in L^{1}(\mathbb{T})$ and we may associate with $f$ the Radon measure $f d \mu$ on $\mathbb{T}$ i.e., a linear functional on $C(\mathbb{T})$ :

$$
C(\mathbb{T}) \ni \varphi \mapsto \int_{\mathbb{T}} f \varphi d \mu
$$

We use this observation in the following lemma.

Lemma 6.8 Suppose $W \in \mathcal{R}(\mathbb{T})$ and for any small $\varepsilon>0$ the function $f_{\varepsilon}=f_{\varepsilon}(x)$ satisfies

$$
f_{\varepsilon}=\pi_{Y} f_{\varepsilon} \in L^{2}(\mathbb{T}), \quad\left\|f_{\varepsilon}\right\|^{2}=1, \quad Y=(a-\varepsilon, a+\varepsilon) .
$$

Let $L_{a}$ be determined by (2.9). Then there exists the weak limi

$$
\lim _{\varepsilon \searrow 0}\left|L_{a} * f_{\varepsilon}\right|^{2}=\nu, \quad \nu=\nu(x, a)=\sum_{m \in \mathbb{Z}} v_{m}(a) e^{i m(x-a)} .
$$

\footnotetext{
${ }^{8}$ i.e., we regard here $\left|L_{a} * f_{\varepsilon}\right|$ as functionals on $C(\mathbb{T})$
} 
The limit (6.21) is independent of the choice of the family $f_{\varepsilon}$, satisfying (6.20). For any $a \in \mathbb{T}$

$$
d \widetilde{\mu}_{W, a}=\frac{1}{2 \pi} \nu(x, a) d x
$$

is a (non-negative) measure on $\mathbb{T}$ and its norm as a functional on $C(\mathbb{T})$ satisfies

$$
\left\|\widetilde{\mu}_{W, a}\right\|_{C^{*}}=\int_{\mathbb{T}} d \widetilde{\mu}_{W, a} \leq \mathbf{c}^{2}=\|W\|_{\mathcal{D} \mathcal{T}}^{2} .
$$

Remark 6.1 By using (4.4) we obtain the equation

$$
\nu(x, a)=\sum_{m, n \in \mathbb{Z}} \omega_{m, n} e^{i m x+i n a} .
$$

Corollary 6.2 Suppose $W \in \mathcal{R}(\mathbb{T})$. Then by (4.9) we have $\|W\|_{\mu}^{2}=\mathbf{T}(W)=\omega_{0,0}$. Therefore by (6.24)

$$
\|W\|_{\mu}^{2}=\int_{\mathbb{T}^{2}} \nu(x, a) d a d x .
$$

Proof of Lemma 6.8. For any $f_{\varepsilon}$ satisfying (6.20)

$$
\left.\left|\frac{1}{2 \pi} \int_{\mathbb{T}} e^{-i k x}\right| L_{a} * f_{\varepsilon}\right|^{2} d x \mid \leq\left\|\operatorname{Conv}_{L_{a}}\right\|^{2} \leq \mathbf{c}^{2}, \quad k \in \mathbb{Z} .
$$

Hence absolute values of Fourier coefficients of any function $\nu_{\varepsilon}(a, \cdot)=\left|L_{a} * f_{\varepsilon}\right|^{2}$ do not exceed $\mathbf{c}^{2}$. Existence of the weak limit (6.21) in the class of distributions with bounded Fourier coefficients is equivalent to the existence of limits for all Fourier coefficients:

$$
\xi_{m}=\lim _{\varepsilon \searrow 0} \xi_{\varepsilon, m}, \quad \xi_{\varepsilon, m}=\frac{1}{2 \pi} \int_{\mathbb{T}} e^{-i m x}\left|L_{a} * f_{\varepsilon}\right|^{2} d x,
$$

independence of these limits of $f_{\varepsilon}$, and the equation $\xi_{m}=e^{-i m a} v_{m}(a)$. The convergence (6.26) does not need to be uniform in $m$.

We put $f_{\varepsilon}=\sum_{k} f_{\varepsilon, k} e^{i k x}$. Below for brevity we skip the subscript $\varepsilon$ and write $f_{k}$ instead of $f_{\varepsilon, k}$. Then $L_{a} * f_{\varepsilon}=\sum_{k} w_{k}(a) f_{k} e^{i k x}$ and

$$
\xi_{\varepsilon, m}=\sum_{k \in \mathbb{Z}} w_{k+m}(a) \bar{w}_{k}(a) f_{k+m} \bar{f}_{k} .
$$

By (6.25) $\left|\xi_{\varepsilon, m}\right| \leq \mathbf{c}^{2}$ for any $m \in \mathbb{Z}$.

Now we show that for any $m \in \mathbb{Z}$ convergence (6.26) takes place. We put

$$
\widetilde{\xi}_{\varepsilon, m}=\sum_{k \in \mathbb{Z}} \sum_{\left|l^{\prime}\right|,\left|l^{\prime \prime}\right| \leq B} w_{k+m}(a) \bar{w}_{k}(a) \frac{f_{k+m+l^{\prime}} \bar{f}_{k+l^{\prime \prime}}}{(2 B+1)^{2}} e^{i\left(l^{\prime}-l^{\prime \prime}\right) a} .
$$


Then by (2.10) and (2.5)

$$
\begin{aligned}
\left|\xi_{\varepsilon, m}-\widetilde{\xi}_{\varepsilon, m}\right| & \leq \sum_{k \in \mathbb{Z}} \sum_{\left|l^{\prime}\right|,\left|l^{\prime \prime}\right| \leq B}\left|w_{k+m}(a) \bar{w}_{k}(a)\right| \frac{\left|f_{k+m} \bar{f}_{k}-f_{k+m+l^{\prime}} \bar{f}_{k+l^{\prime \prime}} e^{i\left(l^{\prime}-l^{\prime \prime}\right) a}\right|}{(2 B+1)^{2}} \\
& \leq \sum_{k \in \mathbb{Z}\left|l^{\prime}\right|,\left|l^{\prime \prime}\right| \leq B} \mathbf{c}^{2} \frac{\left|f_{k+m}-f_{k+m+l^{\prime}} e^{i l^{\prime} a}\right| \cdot\left|f_{k}\right|+\left|f_{k+m+l^{\prime}}\right| \cdot\left|f_{k}-f_{k+l^{\prime \prime}} e^{i l^{\prime \prime} a}\right|}{(2 B+1)^{2}} \\
& \leq \frac{2 \mathbf{c}^{2}}{2 B+1} \sum_{|l| \leq B}\left\|f_{\varepsilon}\right\| \cdot\left\|f_{\varepsilon}-e^{-i l(x-a)} f_{\varepsilon}\right\| \\
& \leq \frac{2 \mathbf{c}^{2}}{2 B+1}\left\|f_{\varepsilon}\right\|^{2} \sum_{|l| \leq B} \frac{\varepsilon^{3 / 2}}{\sqrt{\pi}}|l| \leq 2 \varepsilon^{3 / 2} B \mathbf{c}^{2}\left\|f_{\varepsilon}\right\|^{2} .
\end{aligned}
$$

By using in (6.27) the notation $l=l^{\prime}, n=k+l^{\prime}$, and $s=l^{\prime}-l^{\prime \prime}$, we obtain:

$$
\widetilde{\xi}_{\varepsilon, m}=\sum_{n \in \mathbb{Z}} \sum_{|l|,|s-l| \leq B} w_{n+m-l}(a) \bar{w}_{n-l}(a) \frac{f_{n+m} \bar{f}_{n-s}}{(2 B+1)^{2}} e^{i s a} .
$$

If we fix $n \in \mathbb{Z}$ and $s \in[-2 B, 2 B] \cap \mathbb{Z}$ in the last sum then $n-l \in I_{n, s}$, where

$$
I_{n, s}= \begin{cases}{[n-B, n+B-s] \cap \mathbb{Z}} & \text { if } s \geq 0 \\ {[n-B-s, n+B] \cap \mathbb{Z}} & \text { if } s<0\end{cases}
$$

We also put

$$
b_{m}=\frac{2 B+1-|m|}{(2 B+1)^{2}}, \quad|m| \leq 2 B+1
$$

Then $\sum_{|s| \leq 2 B} b_{s}=1$. By (4.3)

$$
\widetilde{\xi}_{\varepsilon, m}=\sum_{k \in \mathbb{Z},|s| \leq 2 B} v_{I_{k, s}, m}(a) b_{s} f_{k+m} \bar{f}_{k-s} e^{i s a} .
$$

Since $W$ is regular, then by Lemma 4.1 for any $m \in \mathbb{Z}$ and any $\sigma>0$ there exists $Q_{m}=Q_{m}(\sigma)$ such that

$$
\# I \geq Q_{m} \quad \text { implies }\left|v_{I, m}(a)-v_{m}(a)\right|<\sigma .
$$

Then $\widetilde{\xi}_{\varepsilon, m}=\Sigma_{1}+\Sigma_{2}+\Sigma_{3}$, where

$$
\begin{aligned}
\Sigma_{1} & =\sum_{k \in \mathbb{Z},|s| \leq 2 B} v_{m}(a) b_{s} f_{k+m} \bar{f}_{k-s} e^{i s a}, \\
\Sigma_{2} & =\sum_{k \in \mathbb{Z}, 2 B+1-|s| \geq Q_{m}}\left(v_{I_{k, s}, m}(a)-v_{m}(a)\right) b_{s} f_{k+m} \bar{f}_{k-s} e^{i s a}, \\
\Sigma_{3} & =\sum_{k \in \mathbb{Z}, 2 B+1-|s|<Q_{m}}\left(v_{I_{k, s}, m}(a)-v_{m}(a)\right) b_{s} f_{k+m} \bar{f}_{k-s} e^{i s a} .
\end{aligned}
$$


By using Lemma 2.1, we obtain:

$$
\begin{aligned}
\left|\Sigma_{1}-e^{-i m a} v_{m}(a)\left\|f_{\varepsilon}\right\|^{2}\right| & =\left|\sum_{|s| \leq 2 B} e^{-i m a} v_{m}(a) b_{s} \sum_{k \in \mathbb{Z}}\left(f_{k+m} \bar{f}_{k-s} e^{i(m+s) a}-\left|f_{k}\right|^{2}\right)\right| \\
& \leq 3 \mathbf{c}^{2}(2 B+|m|) \varepsilon\left\|f_{\varepsilon}\right\|^{2} .
\end{aligned}
$$

If $2 B+1-|s| \geq Q_{m}$ then by using (6.29) we can estimate $\Sigma_{2}$ :

$$
\left|\Sigma_{2}\right| \leq \sum_{k \in \mathbb{Z}, 2 B+1-|s| \geq Q_{m}} \sigma b_{s} f_{k+m} \bar{f}_{k-s} \leq \sigma\|f\|^{2}
$$

We have:

$$
\begin{aligned}
\left|\Sigma_{3}\right| & \leq 2 \mathbf{c}^{2} \sum_{k \in \mathbb{Z}, 2 B+1-|s|<Q_{m}} b_{s}\left|f_{k+m} \bar{f}_{k-s}\right| \\
& \leq 2 \mathbf{c}^{2} \sum_{2 B+1-|s|<Q_{m}} b_{s}\left\|f_{\varepsilon}\right\|^{2} \leq 2 \mathbf{c}^{2} \frac{\left(1+Q_{m}\right) Q_{m}}{(2 B+1)^{2}}\left\|f_{\varepsilon}\right\|^{2} .
\end{aligned}
$$

Combining estimates (6.28), (6.30), (6.31), and (6.32), we obtain:

$$
\left|\xi_{\varepsilon, m}-e^{-i m a} v_{m}(a)\left\|f_{\varepsilon}\right\|^{2}\right| \leq\left(\left(2 \varepsilon^{3 / 2} B+6 \varepsilon B+3 \varepsilon|m|\right) \mathbf{c}^{2}+\sigma+2 \mathbf{c}^{2} \frac{\left(1+Q_{m}\right) Q_{m}}{(2 B+1)^{2}}\right)\left\|f_{\varepsilon}\right\|^{2} .
$$

This implies existence of the limits (6.26). Since the functions $\nu_{\varepsilon}(a, \cdot)$ are nonnegative, $d \widetilde{\mu}_{W, a}$ is a measure on $\mathbb{T}$ for any $a \in \mathbb{T}$.

\subsection{The space $\mathcal{A C F}(\mathbb{T})$}

Definition 6.2 We say that $f \in L^{\infty}(\mathbb{T})$ lies in the space $\mathcal{A C F}(\mathbb{T})$ if it has absolutely converging Fourier series.

For any $f \in \mathcal{A C \mathcal { F }}(\mathbb{T})$ we put

$$
\|f\|_{\mathcal{D} \mathcal{T}}=\sum_{k \in \mathbb{Z}}\left|f_{k}\right|
$$

Then for any $f \in \mathcal{A C F}(\mathbb{T})$ we have: $\|f\|_{\mathcal{D} \mathcal{T}}=\|\widehat{f}\|_{\mathcal{D} \mathcal{T}}$ and the estimate (2.8). Assertion (14) implies the following

Corollary 6.3 The space $\left(\mathcal{A C \mathcal { F }}(\mathbb{T}),\|\cdot\|_{\mathcal{D T}}\right)$ is a commutative $\mathbb{C}^{*}$-subalgebra in the $\mathbb{C}^{*}$ algebra $\left(\mathcal{D} \mathcal{T}(\mathbb{T}),\|\cdot\|_{\mathcal{D} \mathcal{T}}\right)$.

Suppose $\nu$ satisfies (6.24) for some $W \in \mathcal{R}(\mathbb{T})$. Consider the functions

$$
\varphi=\frac{1}{2 \pi} \int_{\mathbb{T}} \nu(x, \cdot) d x \quad \text { and } \quad \psi=\frac{1}{2 \pi} \int_{\mathbb{T}} \nu(\cdot, a) d a
$$


Lemma 6.9 Suppose $W \in \mathcal{R}(\mathbb{T})$. Then $\varphi, \psi \in \mathcal{A C F}(\mathbb{T})$. Moreover,

$$
\|\varphi\|_{\mathcal{D} \mathcal{T}} \leq\|W\|_{\mathcal{D} \mathcal{T}}^{2}, \quad\|\psi\|_{\mathcal{D} \mathcal{T}} \leq\|W\|_{\mathcal{D} \mathcal{T}}^{2}
$$

Proof. By (6.24)

$$
\frac{1}{2 \pi} \int_{\mathbb{T}} \nu(x, a) d x=\sum_{n \in \mathbb{Z}} \omega_{0, n} e^{i n a} .
$$

By $(10.21) \sum\left|\omega_{0, n}\right| \leq\|W\|_{\mathcal{D} \mathcal{T}}^{2}$.

The case of the function $\psi$ is analogous.

\subsection{The operator $\widehat{g}_{1} W \widehat{g}_{2}$}

Proposition 6.1 Suppose $W \in \mathcal{R}(\mathbb{T}), g_{2} \in L^{\infty}(\mathbb{T})$, and $g_{1},\left|g_{2}\right|^{2} \in \mathcal{A C F}(\mathbb{T})$. Then the operator $\widetilde{W}=\widehat{g}_{1} W \widehat{g}_{2}$ is also regular and the corresponding coefficients $\widetilde{\omega}_{m, n}$ satisfy

$$
\sum_{m, n \in \mathbb{Z}} \widetilde{\omega}_{m, n} e^{i m x+i n a}=\left|g_{1}(x)\right|^{2} \nu(x, a)\left|g_{2}(a)\right|^{2} .
$$

Corollary 6.4 Suppose $W \in \mathcal{R}(\mathbb{T}), g_{2} \in L^{\infty}(\mathbb{T})$, and $g_{1},\left|g_{2}\right|^{2} \in \mathcal{A C F}(\mathbb{T})$. Then by (4.2) and 4.9 )

$$
\left\|\widehat{g}_{1} W \widehat{g}_{2}\right\|_{\mu}^{2}=\frac{1}{(2 \pi)^{2}} \int_{\mathbb{T}^{2}}\left|g_{1}(x)\right|^{2} \nu(x, a)\left|g_{2}(a)\right|^{2} d a d x .
$$

Proposition 6.1 follows from Lemmas 8.1 and 8.2 while Corollary 6.4 is a combination of Corollaries 8.1 and 8.2 .

We associate with any $W \in \mathcal{R}(\mathbb{T})$ the measure $\mu_{W}$ on $\mathcal{X} \times \mathcal{X}$ :

$$
d \mu_{W}=\nu(x, a) \frac{d x d a}{4 \pi^{2}} .
$$

\section{A bistochastic operator generated by $\mu_{W}$}

Lemma 7.1 Suppose $W$ satisfies (at least) one of the following conditions:

(1) $W=U_{F}$, where $F \in \operatorname{Aut}(\mathcal{X}, \mu)$,

(2) $W$ is an operator on $\mathcal{H}=L^{2}(\mathcal{X}), \mathcal{X}=\mathbb{Z}_{J}$,

(3) $W \in \mathcal{R}(\mathbb{T})$.

Then the corresponding measure $\mu_{W}$ determines a bounded operator

$$
\mathcal{W}: L^{1}(\mathcal{X}, \mu) \rightarrow L^{1}(\mathcal{X}, \mu), \quad f \mapsto \mathcal{W} f=\int_{\mathcal{X}} \nu(\cdot, a) f(a) d \mu(a) .
$$

This operator satisfies the estimate

$$
\|\mathcal{W}\|_{L^{1} \rightarrow L^{1}} \leq\|W\|_{\mathcal{D} \mathcal{T}}^{2}
$$

and moreover, has the following properties 
(a). $\mathcal{W}$ is nonnegative: $\mathcal{W} f \geq 0$ whenever $0 \leq f \in L^{1}(\mathcal{X}, \mu)$.

If $W$ is unitary 9 then two more statements hold.

(b). $\mathcal{W} \mathbf{1}_{\mathcal{X}}=\mathbf{1}_{\mathcal{X}}$.

(c). $\int_{\mathcal{X}} \mathcal{W} f(x) d \mu(x)=\int_{\mathcal{X}} f(a) d \mu(a)$ for any $f \in L^{1}(\mathcal{X}, \mu)$.

Conditions (a)-(c) mean that $\mathcal{W}$ is a bistochastic (doubly stochastic) operator.

Proof of Lemma 7.1. (1) In this case $d \mu_{W}(x, a)=\delta(x, F(a)) d \mu(x) d \mu(a)$ (see (6.9)). Then by (6.5) - 6.7) for any $f \in L^{1}(\mathcal{X})$

$$
\mathcal{W} f(x)=\int_{\mathcal{X}} \delta(x, F(a)) f(a) d \mu(a)=\int_{\mathcal{X}} \delta\left(F^{-1}(x), a\right) f(a) d \mu(a)=f \circ F^{-1}(x) .
$$

Hence, $\|\mathcal{W} f\|_{1}=\|f\|_{1}$. Therefore $\mathcal{W}$ is an isometry and condition (c) holds. Conditions (a) and (b) also follow from (7.2).

(2) If $\mathcal{X}=\mathbb{Z}_{J}$ then by (6.13) we have:

$$
f(a)=\sum_{j \in \mathbb{Z}} f_{j} \eta^{j a}, \quad \mathcal{W} f(x)=\sum_{m, n \in \mathbb{Z}} \omega_{m,-n} f_{n} \eta^{m x}
$$

Hence, by (6.17)

$$
\|\mathcal{W} f\|_{1} \leq \sum_{m, n \in \mathbb{Z}_{J}}\left|\omega_{m,-n} f_{n}\right| \leq\|W\|_{\mathcal{D} \mathcal{T}}^{2}\|f\|_{1}
$$

Condition (a) follows from Lemma 6.6, while Conditions (b) and (c) from Lemma 6.7.

(3) In this case for any $f \in L^{1}(\mathbb{T})$

$$
\|\mathcal{W} f\|_{1}=\frac{1}{(2 \pi)^{2}} \int_{\mathbb{T}} d x \int_{\mathbb{T}}|\nu(x, a) f(a)| d a=\frac{1}{2 \pi} \int_{\mathbb{T}} \varphi(a)|f(a)| d a,
$$

where $\varphi$ is determined by (6.33). By Lemma 6.9 $\|\varphi\|_{\infty} \leq\|\varphi\|_{\mathcal{D} \mathcal{T}} \leq\|W\|_{\mathcal{D} \mathcal{T}}^{2}$. This implies (7.1).

To prove (a), we fix $0 \leq f \in L^{1}(\mathbb{T})$ and take any nonnegative continuous function $g: \mathbb{T} \rightarrow \mathbb{R}$. Then $\frac{1}{2 \pi} \int_{\mathbb{T}} g(x) \mathcal{W} f(x) d x$ is the result of the action of the measure $\nu$ on the non-negative function $g(x) f(a)$. This result is nonnegative. Hence, $\mathcal{W} f \geq 0$.

Now suppose $W$ is unitary. Then by (6.35) for any $0 \not \equiv g \in \mathcal{A C \mathcal { F }}(\mathbb{T})$

$$
\frac{1}{2 \pi} \int_{\mathbb{T}}|g(x)|^{2} \mathcal{W} \mathbf{1}_{\mathbb{T}}(x) d x=\frac{1}{(2 \pi)^{2}} \int_{\mathbb{T}^{2}}|g(x)|^{2} \nu(x, a) d x d a=\|\widehat{g} W\|_{\mu}^{2}=\|\widehat{g}\|_{\mu}^{2}
$$

(the last equation follows from Corollary 4.1). The equation

$$
\|\widehat{g}\|_{\mu}^{2}=\|g\|^{2}=\frac{1}{2 \pi} \int_{\mathbb{T}}|g(x)|^{2} d x
$$

implies (b).

\footnotetext{
${ }^{9}$ In case (1) this condition automatically holds.
} 
It is sufficient to check condition (c) only for $f \geq 0$. First, consider the case when $0 \leq f \in L^{\infty}(\mathbb{T})$. Consider $g \geq 0$ such that $f=g^{2}$. Then by Corollary 6.4

$$
\frac{1}{2 \pi} \int_{\mathbb{T}} \mathcal{W} f(x) d x=\frac{1}{(2 \pi)^{2}} \int_{\mathbb{T}^{2}} \nu(x, a)|g(a)|^{2} d x d a=\|W \widehat{g}\|_{\mu}^{2}=\|\widehat{g}\|_{\mu}^{2}=\frac{1}{2 \pi} \int_{\mathbb{T}} f(a) d a .
$$

If $0 \leq f \in L^{1}(\mathbb{T})$ is unbounded, by using a cut off, for any $\varepsilon>0$ we have: $f=f_{1}+f_{2}$, $0 \leq f_{1} \in L^{\infty}(\mathbb{T}),\left\|f_{2}\right\|_{1}<\varepsilon$. Then by (7.1)

$$
\left\|\mathcal{W} f-\mathcal{W} f_{1}\right\|_{1}<\|W\|_{\mathcal{D} \mathcal{T}}^{2} \varepsilon
$$

This implies

$$
\left|\frac{1}{2 \pi} \int_{\mathbb{T}} \mathcal{W} f(x) d x-\frac{1}{2 \pi} \int_{\mathbb{T}} \mathcal{W} f_{1}(x) d x\right| \leq\|W\|_{\mathcal{D} \mathcal{T}}^{2} \varepsilon
$$

The estimate

$$
\frac{1}{2 \pi} \int_{\mathbb{T}} \mathcal{W} f_{1}(x) d x=\frac{1}{2 \pi} \int_{\mathbb{T}} f_{1}(x) d x=\frac{1}{2 \pi} \int_{\mathbb{T}} f(x) d x+\Delta, \quad|\Delta| \leq \varepsilon
$$

finishes the proof.

\section{Product with $\widehat{g}$}

In this section we prove Proposition 6.1 and Corollary 6.4.

\subsection{The operator $W \widehat{g}$}

Lemma 8.1 Suppose $W \in \mathcal{R}(\mathbb{T}), g \in L^{\infty}(\mathbb{T})$, and $|g|^{2} \in \mathcal{A C F}(\mathbb{T})$. Then $\widetilde{W}=W \widehat{g}$ is also regular and the corresponding coefficients $\widetilde{\omega}_{m, n}$ satisfy

$$
\sum_{m, n \in \mathbb{Z}} \widetilde{\omega}_{m, n} e^{i m x+i n a}=\nu(x, a)|g(a)|^{2} .
$$

Corollary 8.1 Suppose $W \in \mathcal{R}(\mathbb{T})$ and $g \in \mathcal{A C F}(\mathbb{T})$. Then by (4.2) and 4.9)

$$
\|W \widehat{g}\|_{\mu}^{2}=\frac{1}{(2 \pi)^{2}} \int_{\mathbb{T}^{2}} \nu(x, a)|g(a)|^{2} d a d x .
$$

Proof of Lemma 8.1. We denote by $\widetilde{\omega}_{I, m, n}$ the quantities (4.1), corresponding to the operator $\widetilde{W}=W \widehat{g}$. Then

$$
\widetilde{\omega}_{I, m, n}=\frac{1}{\# I} \sum_{k, s, j \in \mathbb{Z}, l \in I} W_{l+m, k} g_{k-j} \bar{W}_{l, s} \bar{g}_{s-j-n}=\frac{1}{\# I} \sum_{k, q \in \mathbb{Z}, l \in I} W_{l+m, k} \bar{W}_{l, k-q+n} \mathbf{g}_{q},
$$

where $\mathbf{g}_{q}=\sum_{p \in \mathbb{Z}} g_{p} \bar{g}_{p-q}$. Note that $\mathbf{g}_{q}$ is the $q$-th Fourier coefficient of the function $|g|^{2}$. Indeed,

$$
\mathbf{g}_{q}=\left\langle g, g e^{i q x}\right\rangle=\frac{1}{2 \pi} \int_{\mathbb{T}}|g(x)|^{2} e^{-i q x} d x .
$$


By assumption of the lemma the series $\sum \mathbf{g}_{q}$ absolutely converges. We put

$$
\sum_{q \in \mathbb{Z}}\left|\mathbf{g}_{q}\right|=\mathbf{c}_{g}
$$

For any $\sigma>0$ there exists $Q(\sigma) \in \mathbb{N}$ such that $\sum_{|q|>Q(\sigma)}\left|\mathbf{g}_{q}\right|<\sigma$. Given a small $\sigma$ we have: $\widetilde{\omega}_{I, m, n}=\Omega_{1}+\Omega_{2}$, where

$$
\begin{aligned}
& \Omega_{1}=\frac{1}{\# I} \sum_{k \in \mathbb{Z}, l \in I,|q| \leq Q(\sigma)} W_{l+m, k} \bar{W}_{l, k-q+n} \mathbf{g}_{q}, \\
& \Omega_{2}=\frac{1}{\# I} \sum_{k \in \mathbb{Z}, l \in I,|q|>Q(\sigma)} W_{l+m, k} \bar{W}_{l, k-q+n} \mathbf{g}_{q} .
\end{aligned}
$$

In the sum $\Omega_{1}$ the index $q$ contains only a finite number of values. Hence for all sufficiently big \#I we have:

$$
\Omega_{1}=\sum_{|q| \leq Q(\sigma)} \omega_{m, n-q} \mathbf{g}_{q}+\Delta, \quad|\Delta| \leq \sum_{|q| \leq Q(\sigma)} \sigma\left|\mathbf{g}_{q}\right| \leq \sigma \mathbf{c}_{g} .
$$

Now we estimate $\Omega_{2}$. Let $c_{k}$ be the majorating sequence for $W$ with $\sum c_{k}=\mathbf{c}$. Then

$$
\begin{aligned}
\left|\Omega_{2}\right| & \leq \frac{1}{\# I} \sum_{k \in \mathbb{Z}, l \in I|q|>Q(\sigma)} c_{l+m-k} c_{l-k+q-n}\left|\mathbf{g}_{q}\right| \\
& \leq \sum_{j \in \mathbb{Z},|q|>Q(\sigma)} c_{m+j} c_{q-n+j}\left|\mathbf{g}_{q}\right|=\sum_{|q|>Q(\sigma)} \widetilde{c}_{m+n-q} \sigma\left|\mathbf{g}_{q}\right|
\end{aligned}
$$

where $\widetilde{c}_{s}=\sum_{j} c_{s+j} c_{j}, \quad \sum_{s} \widetilde{c}_{s}=\mathbf{c}^{2}$. Hence

$$
\left|\Omega_{2}\right| \leq \mathbf{c}^{2} \sum_{|q|>Q(\sigma)}\left|\mathbf{g}_{q}\right| \leq \mathbf{c}^{2} \sigma
$$

By (10.2)

Therefore

$$
\left|\sum_{|q|>Q(\sigma)} \omega_{m, n-q} \mathbf{g}_{q}\right| \leq \mathbf{c}^{2} \sum_{|q|>Q(\sigma)}\left|\mathbf{g}_{q}\right| \leq \mathbf{c}^{2} \sigma
$$

$$
\left|\widetilde{\omega}_{I, m, n}-\sum_{q \in \mathbb{Z}} \omega_{m, n+q} \mathbf{g}_{q}\right| \leq \sigma\left(\mathbf{c}_{g}+2 \mathbf{c}^{2}\right) .
$$

Finally note that

$$
\begin{aligned}
\nu(x, a)|g(a)|^{2} & =\sum_{q, p \in \mathbb{Z}} g_{p} \bar{g}_{p-q} e^{i q a} \sum_{m, k \in \mathbb{Z}} \omega_{m, k} e^{i m x+i k a} \\
& =\sum_{m, n, q, p \in \mathbb{Z}} \omega_{m, n-q} g_{p} \bar{g}_{p-q} e^{i m x+i n a} .
\end{aligned}
$$

These computations imply that for all sufficiently big $\# I$ the quantity $\widetilde{\omega}_{I, m, n}$ differs arbitrarily small from

$$
\sum_{p, q \in \mathbb{Z}} \omega_{m, n+q} g_{p} \bar{g}_{p-q}=\frac{1}{(2 \pi)^{2}} \int_{\mathbb{T}^{2}} e^{-i m x-i n a} \nu(x, a)|g(a)|^{2} d x d a .
$$




\subsection{The operator $\widehat{g} W$}

Lemma 8.2 Suppose $W \in \mathcal{R}(\mathbb{T})$ and $g \in \mathcal{A C F}(\mathbb{T})$. Then $\widetilde{W}=\widehat{g} W$ is also regular and the corresponding coefficients $\widetilde{\omega}_{m, n}$ satisfy

$$
\sum_{m, n \in \mathbb{Z}} \widetilde{\omega}_{m, n} e^{i m x+i n a}=|g(x)|^{2} \nu(x, a)
$$

Corollary 8.2 Suppose $W \in \mathcal{R}(\mathbb{T})$ and $g \in \mathcal{A C F}(\mathbb{T})$. Then by (4.2) and (4.9)

$$
\|\widehat{g} W\|_{\mu}^{2}=\frac{1}{(2 \pi)^{2}} \int_{\mathbb{T}^{2}}|g(x)|^{2} \nu(x, a) d x d a
$$

Proof of Proposition 8.2. We denote by $\widetilde{\omega}_{I, m, n}$ the quantities (4.1), corresponding to the operator $\widetilde{W}=\widehat{g} W$. Then

$$
\begin{aligned}
\widetilde{\omega}_{I, m, n} & =\frac{1}{\# I} \sum_{k, s, j \in \mathbb{Z}, l \in I} g_{l+m-k} W_{k, j} \bar{g}_{l-s} \bar{W}_{s, j+n} \\
& =\frac{1}{\# I} \sum_{k, s, j \in \mathbb{Z}, l \in I} g_{m-k} \bar{g}_{-s} W_{k+l, j} \bar{W}_{s+l, j+n} \\
& =\frac{1}{\# I} \sum_{k, s, j \in \mathbb{Z}, l \in I+s} g_{m-k} \bar{g}_{-s} W_{k-s+l, j} \bar{W}_{l, j+n} \\
& =\sum_{k, s \in \mathbb{Z}} g_{m-k} \bar{g}_{-s} \omega_{I+s, k-s, n} .
\end{aligned}
$$

We define $\mathbf{c}_{g}=\sum_{j \in \mathbb{Z}}\left|g_{j}\right|$. Given $\sigma>0$ we take $M=M(\sigma)>|m|$ such that

$$
\sum_{|j| \geq(M-|m|) / 2}\left|g_{j}\right|<\sigma
$$

We take $N=N(\sigma, M)$ such that for any $j,|j| \leq M$ and any interval $J \subset \mathbb{Z}, \# J>N$

$$
\left|\omega_{J, j, n}-\omega_{j, n}\right|<\sigma
$$

Then $\widetilde{\omega}_{I, m, n}=\Sigma_{0}+\Sigma_{1}+\Sigma_{2}+\Sigma_{3}$, where

$$
\begin{aligned}
\Sigma_{0} & =\sum_{k, s \in \mathbb{Z}} g_{m-k} \bar{g}_{-s} \omega_{k-s, n}, \\
\Sigma_{1} & =\sum_{|k-s| \geq M} g_{m-k} \bar{g}_{-s} \omega_{k-s, n}, \\
\Sigma_{2} & =-\sum_{|k-s|<M} g_{m-k} \bar{g}_{-s}\left(\omega_{I+s, k-s, n}-\omega_{k-s, n}\right), \\
\Sigma_{3} & =\sum_{|k-s| \geq M} g_{m-k} \bar{g}_{-s} \omega_{I, k-s, n} .
\end{aligned}
$$


First, we transform the sum $\Sigma_{0}$ :

$$
\begin{aligned}
\Sigma_{0} & =\frac{1}{(2 \pi)^{2}} \int_{\mathbb{T}^{2}} e^{-i m x-i n a} \sum_{k, s \in \mathbb{Z}} g_{m-k} \bar{g}_{-s} e^{i(m-k+s) x} \omega_{k-s, n} e^{i(k-s) x+i n a} d x d a \\
& =\frac{1}{(2 \pi)^{2}} \int_{\mathbb{T}^{2}} e^{-i m x-i n a}|g(x)|^{2} \nu(x, a) d x d a .
\end{aligned}
$$

We estimate $\Sigma_{1}$ by using (10.1) and (8.5):

$$
\left|\Sigma_{1}\right| \leq \mathbf{c}^{2} \sum_{|k-s| \geq M}\left|g_{m-k}\right|\left|g_{-s}\right| \leq 2 \mathbf{c}^{2} \sum_{\beta \in \mathbb{Z},|\alpha| \geq(M-|m|) / 2}\left|g_{\alpha}\right|\left|g_{\beta}\right| \leq 2 \mathbf{c}^{2} \mathbf{c}_{g} \sigma .
$$

We have by (8.6):

$$
\left|\Sigma_{2}\right| \leq \sigma \sum_{k, s \in \mathbb{Z}}\left|g_{m-k}\right|\left|g_{-s}\right|=\sigma \mathbf{c}_{g}^{2}
$$

The sum $\Sigma_{3}$ is estimated in the same way as $\Sigma_{1}$ :

$$
\left|\Sigma_{3}\right| \leq \mathbf{c}^{2} \sum_{|k-s| \geq M}\left|g_{m-k}\right|\left|g_{-s}\right| \leq 2 \mathbf{c}^{2} \mathbf{c}_{g} \sigma .
$$

Combining (8.7), (8.8), (8.9), and (8.10), we see that

$$
\left.\left|\widetilde{\omega}_{I, m, n}-\frac{1}{(2 \pi)^{2}} \int_{\mathbb{T}^{2}} e^{-i m x-i n a}\right| g(x)\right|^{2} \nu(x, a) d x d a \mid \leq\left(4 \mathbf{c}^{2} \mathbf{c}_{g}+\mathbf{c}_{g}^{2}\right) \sigma .
$$

This implies (8.3).

\section{Weaker assumptions on $g$}

Unfortunately we do not know if equation (6.35) remains valid for $W \in \mathcal{R}(\mathbb{T})$ and arbitrary $g_{1}, g_{2} \in L^{\infty}(\mathbb{T})$. In this section we present two partial results in this direction. More precisely, we show that equations (8.2) and (8.4) hold for $g$ lying in sets which are larger than declared in Corollaries 8.1 and 8.2 , in particular, for $g$ equal to indicators of intervals.

\subsection{Computation of $\|W \widehat{g}\|_{\mu}$}

Lemma 9.1 Suppose $W \in \mathcal{R}(\mathbb{T})$ and $g \in L^{\infty}(\mathbb{T})$. Then equation (8.2) remains valid.

Proof. For any $\sigma>0$ there exists $g_{*} \in \mathcal{A C \mathcal { F }}(\mathbb{T})$ such that

$$
\left\|g_{*}\right\|_{\infty} \leq\|g\|_{\infty} \quad \text { and } \quad\left\|g-g_{*}\right\|<\sigma
$$

Indeed, it is sufficient to take $\widetilde{g}$ equal to a finite, but sufficiently long part of the Fourier series of $g$, so that $\left|\|g\|^{2}-\|\widetilde{g}\|^{2}\right|<\sigma$. Then we define $g_{*}$ as a cut off of $\widetilde{g}$ :

$$
g_{*}(x)=\left\{\begin{array}{ccc}
\widetilde{g}(x) & \text { if } & |\widetilde{g}(x)| \leq\|g\|_{\infty} \\
\|g\|_{\infty} & \text { if } & \widetilde{g}(x)>\|g\|_{\infty} \\
-\|g\|_{\infty} & \text { if } & \widetilde{g}(x)<-\|g\|_{\infty}
\end{array}\right.
$$


The function $g_{*}(x)$ is Lipschitz. Hence, it lies in $\mathcal{A C F}(\mathbb{T})$.

By using the triangle inequality, (1.5) and Assertion (7), we have:

$$
\left|\|W \widehat{g}\|_{\mu}-\left\|W \widehat{g}_{*}\right\|_{\mu}\right| \leq\left\|W\left(\widehat{g}-\widehat{g}_{*}\right)\right\|_{\mu} \leq\|W\|\left\|\left(\widehat{g}-\widehat{g}_{*}\right)\right\|_{\mu} \leq \sigma\|W\| .
$$

On the other hand, by (8.2)

$$
\begin{aligned}
\left.\left|\left\|W \widehat{g}_{*}\right\|_{\mu}^{2}-\frac{1}{(2 \pi)^{2}} \int_{\mathbb{T}^{2}} \nu(x, a)\right| g(a)\right|^{2} d a d x \mid & =\frac{1}{2 \pi}\left|\int_{\mathbb{T}} \varphi(a)\left(\left|g_{*}(a)\right|^{2}-|g(a)|^{2}\right) d a\right|, \\
\varphi & =\frac{1}{2 \pi} \int_{\mathbb{T}} \nu(x, \cdot) d x .
\end{aligned}
$$

By using the estimate $\|\varphi\|_{\infty} \leq\|\varphi\|_{\mathcal{D} \mathcal{T}} \leq\|W\|_{\mathcal{D} \mathcal{T}}^{2}$ (Lemma 6.9), we obtain:

$$
\left.\left|\left\|W \widehat{g}_{*}\right\|_{\mu}^{2}-\frac{1}{(2 \pi)^{2}} \int_{\mathbb{T}^{2}} \nu(x, a)\right| g(a)\right|^{2} d a d x \mid \leq\|W\|_{\mathcal{D} \mathcal{T}}^{2} \sigma
$$

Hence, Lemma 9.1 is a combination of (9.1) and (9.2).

\subsection{Computation of $\|\widehat{g} W\|_{\mu}$}

Definition 9.1 We say that $g \in L^{\infty}(\mathbb{T})$ is almost $\mathcal{A C F}(\mathbb{T})$ (the notation is $g \in \mathcal{A} \mathcal{A C F}(\mathbb{T})$ ) if there exists a continuous in $\varepsilon \in\left(-\varepsilon_{0}, \varepsilon_{0}\right)$ family of functions $g_{\varepsilon} \in L^{\infty}(\mathbb{T})$ such that the following conditions hold:

A1. $g_{0}=g$,

A2. if $\varepsilon \neq 0$ then $g_{\varepsilon} \in \mathcal{A C F}(\mathbb{T})$,

A3. $\left|g_{\varepsilon}-g\right| \leq\left|g_{\varepsilon}-g_{-\varepsilon}\right|$ if $0 \leq \varepsilon<\varepsilon_{0}$,

A4. $\lim _{\varepsilon \rightarrow 0}\left\|g_{\varepsilon}-g_{-\varepsilon}\right\|=0$.

A typical example of an $\mathcal{A} \mathcal{A C F}(\mathbb{T})$ function is the indicator of an interval (see Lemma 9.3 below).

Lemma 9.2 Suppose $W \in \mathcal{R}(\mathbb{T})$ and $g \in \mathcal{A} \mathcal{A C F}(\mathbb{T})$. Then equation 8.4 remains valid.

Proof. Let $g_{\varepsilon}$ be the family from Definition 0.1 . By $\mathbf{A} \mathbf{3}$ and $\mathbf{A} \mathbf{1}$ for any $\varepsilon \in\left(0, \varepsilon_{0}\right)$ and any $X \in \mathcal{B}$

$$
0 \leq\left\|\left(\widehat{g}_{\varepsilon}-\widehat{g}\right) W \pi_{X}\right\|^{2} \leq\left\|\left(\widehat{g}_{\varepsilon}-\widehat{g}_{-\varepsilon}\right) W \pi_{X}\right\|^{2} .
$$

This inequality implies

$$
0 \leq\left\|\left(\widehat{g}_{\varepsilon}-\widehat{g}\right) W\right\|_{\mu}^{2} \leq\left\|\left(\widehat{g}_{\varepsilon}-\widehat{g}_{-\varepsilon}\right) W\right\|_{\mu}^{2}, \quad \varepsilon \in\left(0, \varepsilon_{0}\right) .
$$

By A2 and (8.4) $\left\|\left(\widehat{g}_{\varepsilon}-\widehat{g}\right) W\right\|_{\mu}^{2} \leq \Delta_{\varepsilon}$,

$$
\Delta_{\varepsilon}=\frac{1}{(2 \pi)^{2}} \int_{\mathbb{T}^{2}}\left|g_{\varepsilon}(x)-g_{-\varepsilon}(x)\right|^{2} \nu(x, a) d x d a \leq\left\|g_{\varepsilon}-g_{-\varepsilon}\right\|^{2}\|\psi\|_{\infty}, \quad \psi=\frac{1}{2 \pi} \int_{\mathbb{T}} \nu(\cdot, a) d a
$$

(see also (6.33) $)$. Note that $\|\psi\|_{\infty} \leq\|\psi\|_{\mathcal{D} \mathcal{T}}$. Hence by $\mathbf{A} 4$ and Lemma 6.9 $\Delta_{\varepsilon} \rightarrow 0$ as $\varepsilon \rightarrow 0$.

By triangle inequality

$$
\left.\left|\left\|\widehat{g}_{\varepsilon} W\right\|_{\mu}^{2}-\frac{1}{(2 \pi)^{2}} \int_{\mathbb{T}^{2}}\right| g(x)\right|^{2} \nu(x, a) d a d x|=|\|\widehat{g} W\|_{\mu}^{2}-\left\|\widehat{g}_{\varepsilon} W\right\|_{\mu}^{2} \leq \Delta_{\varepsilon} .
$$

Hence equation (8.4) follows. 


\subsection{Indicators of intervals}

Lemma 9.3 For any interval $I \subset \mathbb{T}$ the function $\mathbf{1}_{I}$ lies in $\mathcal{A} \mathcal{A C F}$.

Proof. We put $g_{0}=\mathbf{1}_{I}$. For $\varepsilon \in(0,1)$ we define

$$
\begin{aligned}
g_{\varepsilon}(x) & =\max \left\{0,1-\frac{1}{\varepsilon} \operatorname{dist}(x, I)\right\}, \\
g_{-\varepsilon}(x) & =1-\max \left\{0,1+\frac{1}{\varepsilon} \operatorname{dist}(x, \mathbb{T} \backslash I)\right\} .
\end{aligned}
$$

Conditions A1-A4 from Definition 9.1 also obviously hold. Hence $\mathbf{1}_{I} \in \mathcal{A} \mathcal{A C F}$.

\section{Coefficients $\omega_{m, n}$}

In this section $W \in \mathcal{D} \mathcal{T}(\mathbb{T}),\left\{c_{k}\right\}_{k \in \mathbb{Z}}$ is its majorating sequence, and $\mathbf{c}=\sum c_{k}=\|W\|_{\mathcal{D} \mathcal{T}}$.

Lemma 10.1 For any $W \in \mathcal{R}(\mathbb{T})$ and any $m, n \in \mathbb{Z}$

$$
\omega_{m, n}=\bar{\omega}_{-m,-n} .
$$

Proof. This equation follows from the identity $\omega_{I, m, n}=\bar{\omega}_{I+m,-m,-n}$ for any interval $I \subset \mathbb{Z}$

We have the estimates

$$
\begin{aligned}
& \sum_{n \in \mathbb{Z}}\left|\omega_{I, m, n}\right| \leq \frac{1}{\# I} \sum_{j, n \in \mathbb{Z}, l \in I} c_{l+m-j} c_{l-j-n}=\mathbf{c}^{2}, \\
& \sum_{m \in \mathbb{Z}}\left|\omega_{I, m, n}\right| \leq \frac{1}{\# I} \sum_{j, n \in \mathbb{Z}, l \in I} c_{l+m-j} c_{l-j-n}=\mathbf{c}^{2} .
\end{aligned}
$$

These estimates imply the following

Corollary 10.1 If $W$ is regular then (compare with (6.17))

$$
\sum_{n \in \mathbb{Z}}\left|\omega_{m, n}\right| \leq \mathbf{c}^{2}, \quad \sum_{n \in \mathbb{Z}}\left|\omega_{n, m}\right| \leq \mathbf{c}^{2} \quad \text { for any } m \in \mathbb{Z} .
$$

We put

$$
P_{M}(m)=\{n \in \mathbb{Z}:|n+m| \geq 2 M\} .
$$

Lemma 10.2 For any $m \in \mathbb{Z}$

$$
\sum_{n \in P_{M}(m)}\left|\omega_{m, n}\right| \leq 2 \mathbf{c} \sum_{|k| \geq M} c_{k} .
$$


Proof. For any integer interval $I$

$$
\begin{aligned}
\sum_{n \in P_{M}(m)}\left|\omega_{I, m, n}\right| & \leq \frac{1}{\# I} \sum_{j \in \mathbb{Z}, n \in P_{M}(m), l \in I} c_{l-j+m} c_{l-j-n} \\
& =\sum_{k \in \mathbb{Z}, n \in P_{M}(m)} c_{m+n-k} c_{k}=\sum_{k \in \mathbb{Z}, s \in P_{M}(0)} c_{s-k} c_{k} \\
& \leq \sum_{k \in \mathbb{Z},|m| \geq M} c_{m} c_{k}+\sum_{m \in \mathbb{Z},|k| \geq M} c_{m} c_{k}=2 \mathbf{c} \sum_{|k| \geq M} c_{k} .
\end{aligned}
$$

In the limit $\# I \rightarrow \infty$ we obtain (10.3).

\section{References}

[1] Accardi L. Note on quantum dynamical entropies. Reports on Math. Phys. 38 (1996), $457-469$.

[2] Accardi L., Ohya M., and Watanabe N. Dynamical entropy through quantum Markov chain. Open System and Information Dynamics.

[3] Alicki R. and Fannes M. Quantum dynamical systems. Oxford University Press. Oxford, 2001.

[4] Beck C., Graudenz D. Symbolic dynamics of successive quantum-mechanical measurements. Phys. Rev. A 46 (1992) 6265-6276.

[5] Collet P. and Eckmann J.-P. (2006) Concepts and results in chaotic dynamics: a short course. Springer-Verlag, Berlin, Heidelberg.

[6] Connes A., Narnhoffer H., and Thirring W. Comm. Math. Phys. 112 (1987), 691.

[7] Cover T.M. and Thomas J.A. Elements of Information Theory. New York: Wiley, 1991.

[8] Downarowicz T. and Frej B. Measure-theoretic and topological entropy of operators on function spaces. Erg. Th. Dyn. Sys. 25, 2005, 455-481.

[9] Downarowicz T. and Frej B. Doubly stochastic operators with zero entropy. arXiv:1803.07882v1 [math.DS] 21 Mar 2018.

[10] Katok A. and Hasselblatt B. (1995) Introduction to the modern theory of dynamical systems. Encyclopedia of mathematics and its applications. Vol 54. Cambridge University Press, Cambridge.

[11] R. M. Dudley. Real Analysis and Probability (2 ed.). Cambridge University Press. 2002.

[12] M. B. Feldman. A Proof of Lusin's Theorem. American Math. Monthly, 88 (1981), $191-2$. 
[13] Ghys E., Langevin R., and Walczak. Entropie mesurée et partitions de l'unité. C. R. Acad. Sci., Paris. Sér I, 303 (1986), 251-254.

[14] Kollár B. and Koniorczyk M. Entropy rate of message sources driven by quantum walks. Phys. Rev. A 89, (2014) 022338.

[15] Makarov I.I. Dynamical entropy for Markov operators. J. Dynam. Control Systems 6 (1), 2000, 1-11.

[16] K. Maurin. Methods of Hilbert spaces. Warszawa, 1967.

[17] Ohya M. Quantum communications and measurement. 2 (1995), 309.

[18] Ohya M. Foundation of entropy, complexity and fractal in quantum systems. Internatinal congress of Probability Towards 2000, 1996.

[19] Pechukas P. Kolmogorov entropy and quantum chaos. J. Phys. Chem. 86 (1982) 2239-2243.

[20] W. M. Schmidt. Diophantine approximation. Lecture Notes in Mathematics 785. Springer, 1980.

[21] Srinivas M.D. Quantum generalization of Kolmogorov entropy. J. Math. Phys. 19, (1978) 1952-1961.

[22] D. Treschev $\mu$-norm of an operator. Proc. of Steklov Math. Inst. 310, (2020), 262290. 